\title{
Thermal depinning and transverse-field tilting transitions in a planar vortex array pinned by a columnar defect
}

\author{
Leo Radzihovsky \\ Department of Physics, University of Colorado, Boulder, CO 80309
}

(Dated: September 20, 2018)

\begin{abstract}
We study thermal and a transverse magnetic field response of a vortex line array confined to a plane with a single columnar pinning defect. By integrating out "bulk" degrees of freedom away from the columnar defect we reduce this two-dimensional problem to a one-dimensional one, localized on the defect and exhibiting a long-range elasticity along the defect. We show that as a function of temperature, for a magnetic field aligned with the defect this system exhibits a one-dimensional analog of a roughening transition, with a low-temperature "smooth" phase corresponding to a vortex array pinned by the defect, and a high-temperature "rough" phase in which at long scales thermal fluctuations effectively average away pinning by the defect. We also find that in the low-temperature pinned phase, the vortex lattice tilt response to a transverse magnetic field proceeds via a soliton proliferation "transition", governed by an integrable sine-Hilbert equation and analogous to the well-known commensurate-incommensurate transition in sine-Gordon systems. The distinguishing feature here is the long-range nature of the one-dimensional elasticity, leading to a logarithmic soliton energy and interaction. We predict the transverse-field-temperature phase diagram and discuss extension of our results to a bulk vortex array in the presence of a dilute concentration of columnar defects.
\end{abstract}

\section{INTRODUCTION}

\section{A. Background and motivation}

The discovery of high-temperature superconductors almost 20 years ago, among other things, has rekindled interest in the magnetic field $(H)$ - temperature $(T)$ phase diagram of type II superconductors ${ }^{1}$, generating a vigorous scientific activity. As a result, much has been clarified about the nature of equilibrium and nonequilibrium properties of vortex states in the presence of thermal fluctuations, pinning disorder and electrical ("super"-) current $^{2-6}$, leading to a rich phase diagram. In particular, in contrast to a mean-field phase diagram, thermal fluctuations drive a first-order melting of a vortex lattice over a large portion of the phase diagram into a resistive (although with large conductivity and diamagnetic response) vortex liquid ${ }^{7-10}$, that, from the symmetry point of view is qualitatively identical to the normal state. Furthermore, as was first shown by Larkin ${ }^{11,12}$, arbitrarily weak pinning disorder, on sufficiently long Larkin scale (that diverges in the limit of vanishing disorder) always disrupts translational order of the vortex lattice. It has been argued theoretically ${ }^{4,13}$, with a limited experimental support ${ }^{14}$, that in the resulting state, vortices are collectively pinned into a vortex glass characterized by an Edwards-Anderson ${ }^{15}$ like order parameter, exhibiting a vanishing linear mobility and therefore a vanishing linear resistivity.

While the original proposal for the vortex glass state was made in a context of intrinsic, short-range correlated (point) disorder, it was soon appreciated that the beneficiary effects of pinning can be enhanced by introducing artificial pinning centers by, for example, electron and/or heavy ion irradiation, with the latter re- sulting in a forest of columnar pinning defects, that is a particularly effective pinning mechanism ${ }^{16}$. The resulting anisotropic vortex glass was dubbed a Bose-glass ${ }^{17,18}$ because of its mathematical connection with interacting two-dimensional (2D) quantum bosons pinned by a quenched (time-independent) random $2 \mathrm{D}$ potential ${ }^{19}$. This connection allowed understanding many of the properties of the anisotropic vortex glass from the corresponding quantum Bose-glass phase. ${ }^{19}$

One key feature of the anisotropic vortex glass that distinguishes it from the corresponding (putative) isotropic one is the existence of the "transverse" Meissner effect, ${ }^{18}$ namely a vanishing response to a field $H_{\perp}<H_{\perp}^{c}$ applied transversely to columnar defects and vortex lines. This expulsion of the transverse flux density, $B_{\perp}$, that has received considerable experimental ${ }^{20}$ and simulations ${ }^{21}$ support, corresponds to an effectively divergent anisotropic vortex glass tilt modulus ${ }^{22}$, that in the quantum correspondence maps onto a vanishing superfluid density in the Bose-glass phase. The detailed theoretical description of the transverse Meissner effect (as well as other properties of the phase) has been predominantly limited to noninteracting vortex lines ${ }^{18,23}$. Although these are supported by scaling theories ${ }^{18,21,23,24}$ (borrowed from the variable-range hopping theory for electronic systems ${ }^{25}$ ) that do incorporate effects of both disorder and interactions (clearly essential for the very existence of the Bose-glass phase), with the exception of functional RG analysis ${ }^{22}$, a detailed interacting description is limited to simulations. ${ }^{21,26}$ This is not surprising, as a description of strongly interacting random systems is a notoriously difficult (with few exceptions) unsolved problem, whose solution is at the heart of understanding many of the interesting condensed matter phenomena. 
One way to incorporate strong interactions is to approach the problem from the vortex solid (rather than the vortex liquid starting point) pinned by a random potential. Potential difficulties with this approach are a proper incorporation of topological defects (dislocations and disclinations) that tend to proliferate in the presence of quenched disorder and external perturbations.

Recent analytical real-space renormalization group (RG) study ${ }^{27}$ that demonstrated stability of a $3 \mathrm{D}$ weakly disordered random-field XY model to a proliferation of topological defects (vortices), provide a strong argument for the stability of an elastically disordered but topologically ordered vortex Bragg-glass phase postulated and studied in detail by Giamarchi and Le Doussal ${ }^{28}$. These studies therefore give support to treatments of vortex solids ${ }^{13,29-31}$ that ignore the notoriously difficult-to-treat topological defects. Furthermore, even if Bragg-glass is unstable to dislocations, for weak disorder dislocations will be dilute, with physics on scales smaller than their spacing expected to be well-described by the vortex Bragg-glass phenomenology. ${ }^{13,28-31}$

A suppression of topological defects can furthermore be facilitated by a planar confinement of vortices, realized in layered high- $\mathrm{T}_{c}$ superconductors (e.g., BISCCO) ${ }^{32}$ or by artificially prepared multilayers ${ }^{33,34}$, where for a magnetic field directed along the planes, vortices are well localized to $2 \mathrm{D}$. The resulting planar (2D) vortex array pinned by point disorder, where dislocations are excluded by construction is in fact the "toy" model studied by Matthew Fisher ${ }^{13}$, that motivated his original proposal of a vortex glass phase in bulk superconductors.

Motivated by these ingredients, in this paper we study a (1+1)-dimensional vortex array confined to a planar slab of thickness $w$ in a presence of a single planar columnar defect, illustrated in Fig.1. This system was introduced and first studied in great detail in Ref. 35.

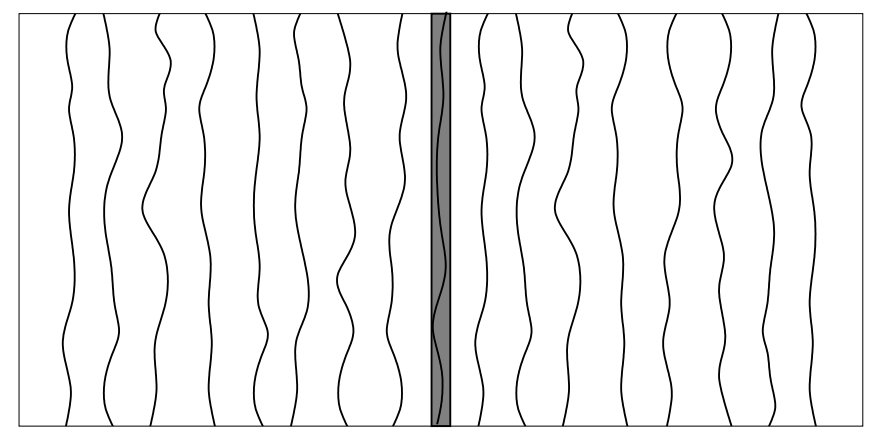

FIG. 1: A (1+1)-dimensional (planar) vortex lattice pinned by a single columnar defect studied in this paper.

As should be clear from the above discussion, such a "toy" model ${ }^{35}$ may be relevant to the regime of farseparated (by $d$, compared to vortex spacing $\sqrt{\phi_{0} / B}$ ) columnar defects, accessible for flux density far exceeding the columnar-defect matching field $B_{\phi}=\phi_{0} / d^{2},{ }^{18,36}$ where $\phi_{0}=h c / 2 e \approx 2.1 \times 10^{-7} \mathrm{G}-\mathrm{cm}^{2}$ is a fundamental quantum of flux. As first investigated in Ref. 35, we study the response of such a $(1+1)$-dimensional vortex array to a planar tilting magnetic field $H_{\perp}$, applied transversely to the columnar defect, as illustrated in Fig.2.

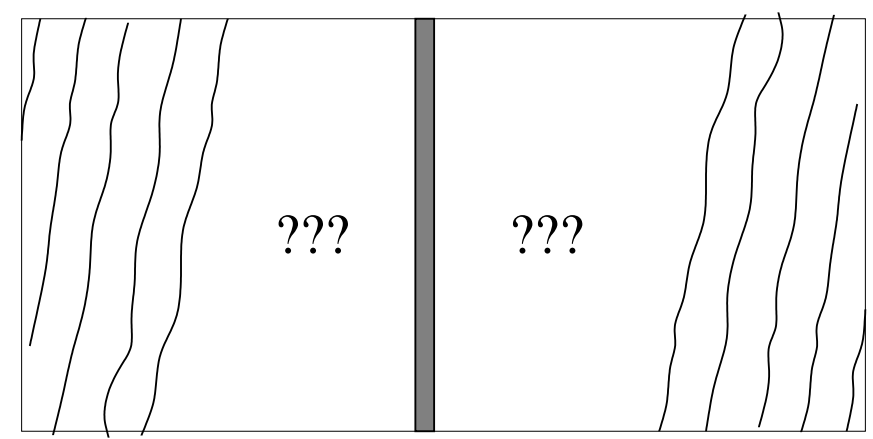

FIG. 2: A (1+1)-dimensional (planar) vortex array induced by a magnetic field $\mathbf{H}$ applied in the plane of the slab, at an angle to a single columnar pinning defect.

The rest of the paper is organized as follows. We conclude the Introduction with a summary of our main results and predictions, heuristically extended to a dilute concentration of columnar defects. In Sec.II we derive the appropriate $(1+1)$-dimensional continuum model for a single defect and discuss its ingredients. By integrating out "bulk" (away from the defect) degrees of freedom we reduce this model to a $(0+1)$-dimensional model confined to a defect, and characterized by a long-range elasticity along the defect. In Sec.III we study the effect of transverse magnetic field $H_{\perp}$ and demonstrate that tilting of the vortex lattice away from a columnar defect proceeds via a novel "commensurate-incommensurate transition" controlled by a proliferation of solitons. In Sec.IV we study effects of thermal fluctuations and demonstrate that this system exhibits a 1D "roughening"-like transition. We explore its consequences for the vortex positional correlations, and construct a $H_{\perp}-T$ phase diagram. We conclude in Sec.V with an extension of these results to an experimentally relevant case of a dilute concentration of columnar defects (allowing for genuine transitions) and close in Sec.VI.with a summary of our study.

\section{B. Summary of results}

The body of the paper is primarily devoted to the study a planar $(1+1)$-dimensional vortex array at a $1 \mathrm{D}$ vortex density $n_{0}=1 / a$, in the presence of thermal fluctuations and induced by an external planar magnetic field $\mathbf{H}=H_{z} \hat{\mathbf{z}}+\mathbf{H}_{\perp}$ applied at an angle to a single columnar defect $^{35}$. As we will show below, even this "toy" problem is quite rich, providing insight into the bulk $(2+1)$ dimensional multi-defect problem. It has the added benefit that it can be analyzed in detail analytically. This is 
not surprising as such a planar classical vortex array is a cousin of a one-dimensional quantum problem, a Luttinger liquid, that is known to be exactly solvable and to exhibit rich phenomenology. ${ }^{37}$ In fact our classical analysis of the vortex problem has strong formal connections to the work of Kane and Fisher ${ }^{38}$ who studied a Luttinger liquid in a presence of a single localized impurity, a problem that admits exact analysis ${ }^{39}$. This connection was first emphasized and fruitfully utilized by Hoffstetter et $a l .{ }^{35}$ and Polkovnikov, et al. ${ }^{40}$, although we will not take advantage of it. As is well known ${ }^{37}$, the Luttinger formalism is equivalent to the classical theory of vortex lattice elasticity that we (and Ref. 35) employ here.

Our work has a strong overlap with that of Ref. 35, particularly on the finite temperature analysis for a vanishing transverse field in an infinite single-pin system, as well as a large transverse field, where tilt response is analytic. Where this overlap exists our predictions are in complete agreement with those found in Ref. 35. However, our emphasis is on the low-temperature, strong coupling regime, where tilt response is highly nonlinear, and can only be understood in detail in terms of vortex lattice solitons, that proliferate at a novel commensurateincommensurate crossover, that, we argue, turns into a genuine sharp phase transition for a dilute concentration of columnar defects.

Hence, as we describe in more detail below, in the presence of a dilute concentration of columnar defects the planar vortex array exhibits two phases in the transverse field $H_{\perp}$ - temperature $T$ phase diagram: a low $T, H_{\perp}$ "commensurate" pinned/aligned (C) phase and a high $T, H_{\perp}$ "incommensurate" depinned/tilted (I) phase. As illustrated in Fig.3, a novel finite temperature commensurate-incommensurate phase transition separates the two phases.

For a vanishing transverse field, the phase transition is akin to a thermal roughening transition ${ }^{41-43}$, that is closely related to a zero-temperature superfluid-insulator transition in a resistively-shunted Josephson junction ${ }^{44}$, the opaque-to-transparent impurity transition in a Luttinger liquid $^{38}$, and spin-boson and dissipative impurity models $^{45-47}$.

Before we discuss our results, a disclaimer is in order here. Because a single columnar defect cannot possibly compete with bulk degrees of freedom, with its effects vanishing in thermodynamic limit, we expect a twodimensional free energy that is analytic, and therefore no true phase transition can take place in a 2D thermodynamic limit. However, as in, for example, the Kondo problem $^{47}$, where one considers the effect of an impurity on the bulk electron gas, bulk effects are predicted only once a finite density of defects is considered. Here too the transitions that we discuss are for the elastic degrees of freedom localized on the columnar defect, that is a boundary critical phenomenon. Although the 1D roughening transition is indeed a genuine one, the contribution of the associated (nonanalytic) free energy to the bulk two-dimensional system vanishes in the $2 \mathrm{D}$ ther-
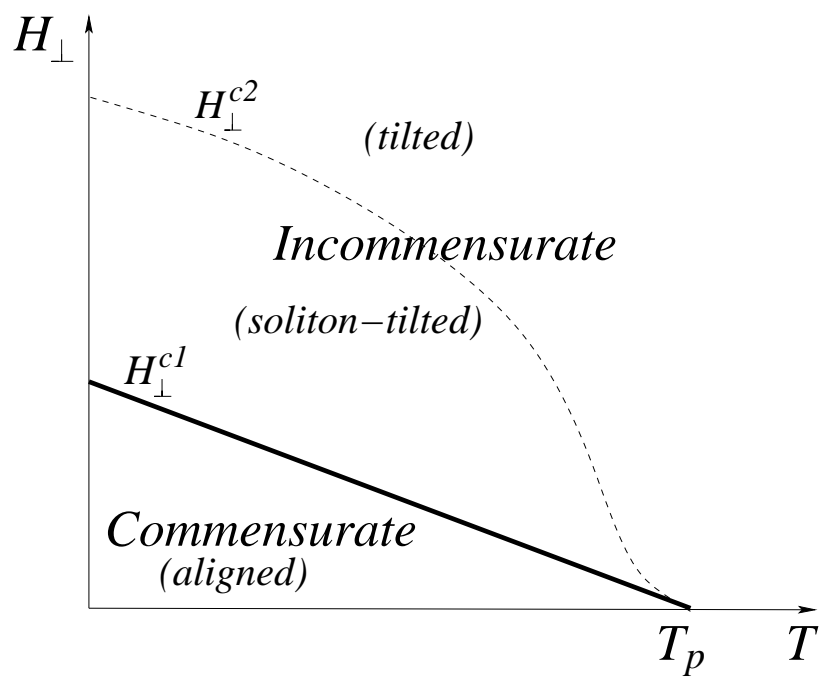

FIG. 3: $H_{\perp}-T$ phase diagram illustrating the commensurate (C) and incommensurate (I) phases separated by a continuous CI phase transition at $H_{\perp}^{c 1}(T)$ (full curve), where vortex lines in the vicinity of far-separated columnar defects tilt via proliferation of solitons. As the soliton density grows with increasing $H_{\perp}$ (dashed curve), the solitons overlap near the upper-critical transverse field $H_{\perp}^{c 2}(T)$, beyond which the system crosses over to a smoothly tilted vortex lattice. The lower-critical field, $H_{\perp}^{c 1}(T)$ vanishes with increasing pin separation $d$, eliminating the CI transition (but not the roughenning transition at $T_{p}$ ) for a single columnar defect.

modynamic limit. Furthermore, the CI (vortex line tilting) "transition" takes place at a lower-critical transverse field $H_{\perp}^{c 1}(L)$, that, in the case of one pin is driven to zero in the thermodynamic limit $(L \rightarrow \infty)$. It only becomes a genuine sharp phase transition for a finite density $1 / d$ of columnar defects, with the lower-critical transverse field $H_{\perp}^{c 1}(d)$ set by the columnar-pin spacing $d{ }^{48}$ Since this latter case is the one most easily accessible experimentally, we heuristically extend our rigorous single pin results to a finite dilute concentration of independent columnar defects. This being a notoriously difficult unsolved problem, we expect this heuristic extension to break down on sufficiently long scales, where collective pinning effects become important. ${ }^{17,18,21-24}$

Our results can be divided into two categories: thermal effects at a vanishing transverse field, i.e., for the magnetic field (and therefore induced planar vortex array) aligned with columnar defects, and a low-temperature response of the vortex array to a tilting (transverse) magnetic field.

For a magnetic field aligned with columnar defects we show that a vortex array undergoes a "roughening"-like transition at

$$
T_{p}=\frac{\sqrt{K B}}{\pi n_{0}^{2}},
$$

between a low-temperature state in which each columnar defect effectively pins the vortex lattice around the 
pin for $T<T_{p}$ and a phase in which its pinning effects, even near a columnar defect, vanish at long scales. In above, $K$ and $B$ are vortex lattice tilt and compressional moduli, whose vortex density and dependence on other parameters (e.g., vortex interaction) can be computed from microscopics $35,43,49,50$. This roughening-like transition is related to a one-dimensional long-range interacting $\left(1 /|i-j|^{2}\right)$ Ising model ${ }^{46}$, that is well-known from a number of other physical contexts, most prominently the Kondo problem ${ }^{47}$, and more recently, in a dissipative Josephson junction ${ }^{44}$ and a Luttinger liquid in the presence of an impurity ${ }^{38}$. In relation to the latter work, we note that unlike the quantum case where there is no simple way to tune the Luttinger parameter ${ }^{51}$, here it can be simply tuned by temperature and vortex density. ${ }^{52}$

As is usually the case for such (e.g., roughening ${ }^{41-43}$, Kosterlitz-Thouless ${ }^{55}$, and other topological) transitions there is no local order parameter and phases are distinguished by long-scale behavior of correlation functions. The low-temperature $T<T_{p}$, pinned phase is characterized by a finite mean-squared vortex fluctuations at the location of the defect (that we take to be $x=0$ ), with correlations away from the defect given by ${ }^{35}$

$$
\left\langle u(z, x) u\left(0, x^{\prime}\right)\right\rangle \approx \frac{k_{B} T}{4 \pi \sqrt{K B}} \ln \left[\frac{K\left(|x|+\left|x^{\prime}\right|\right)^{2}+B z^{2}}{K\left(x-x^{\prime}\right)^{2}+B z^{2}}\right] .
$$

On scales longer the pinning length $\xi$ (defined in Eq.4.10, below), the corresponding average density exhibits Friedel-like oscillations ${ }^{35}$ given by

$$
\langle n(x, z)\rangle_{0} \approx n_{0}+2 n_{G}\left(\frac{\bar{a}}{2|x|}\right)^{\eta / 2} \cos \left(2 \pi n_{0} x\right)
$$

where $\bar{a}=\operatorname{Max}[a, \xi \sqrt{B / K}]$ and

$$
\eta=\frac{2 \pi}{a^{2}} \frac{k_{B} T}{\sqrt{K B}} .
$$

In contrast, at high temperature $T>T_{p}$, vortex thermal fluctuations effectively average away the effects of the pin, leading to phonon correlations that diverge logarithmically with sample size. The connected phonon correlation function is finite and is given by

$$
\left\langle(u(x, z)-u(0,0))^{2}\right\rangle \approx \frac{k_{B} T}{\pi \sqrt{K B}} \ln \left[a^{-1} \sqrt{x^{2}+\frac{B}{K} z^{2}}\right] .
$$

Despite the irrelevance (in the RG sense of the term) of the columnar defect for $T>T_{p}$, the average density also displays perturbative Friedel oscillations ${ }^{35}$

$$
\langle n(x, z)\rangle \approx n_{0}+\frac{n_{G} a v}{k_{B} T} \sqrt{\frac{K}{B}}\left(\frac{a}{|x|}\right)^{\eta-1} \cos \left(2 \pi n_{0} x\right),
$$

with a stronger power-law exponent than that for $T<T_{p}$ and an amplitude that vanishes with the strength of the pinning potential $v$.

The low-temperature $T<T_{p}$, the pinned (commensurate) phase is distinguished from the rough (incommensurate) phase by a transverse Meissner response to a magnetic field $H_{\perp}$ applied transversely to the columnar pin. Namely, we find that for a field smaller than a lower-critical transverse field $H_{c 1}^{\perp}$

$H_{c 1}^{\perp} \approx \frac{\phi_{0}}{w}\left\{\begin{array}{ll}\frac{1}{L} \ln \frac{L}{\xi}, & L \sqrt{\frac{B}{K}} \ll d \\ \sqrt{\frac{B}{K}} \frac{1}{4 \pi d} \ln \left[\sqrt{\frac{K}{B}} \frac{2 \pi d}{\xi}\right], & L \sqrt{\frac{B}{K}} \gg d\end{array}\right.$,

where $L$ is the length of the sample along the columnar defect $(z), w$ is the slab thickness, and $d$ is the columnar pin spacing. At low temperature $\xi \approx \xi_{0}$ is approximately given by

$$
\xi_{0} \approx \frac{a^{2}}{2 \pi^{2}} \frac{\sqrt{B K}}{v} .
$$

For $H^{\perp}<H_{c 1}^{\perp}$ vortex lines in the wide vicinity

$$
\lambda_{h}^{0}=\left(\frac{B}{K}\right)^{1 / 2} \frac{L}{\pi}
$$

of a columnar pin remain aligned with it, therefore exhibiting a bulk transverse Meissner effect for a $d<\lambda_{h}^{0}$ spaced array of pins. Of course, (as for the thermallydriven depinning transition discussed above, here too), because a single pin cannot compete with the bulk magnetic energy, away from the defect beyond this screening length $\lambda_{h}^{0}$ the vortex lattice is always aligned along the applied magnetic field. Related to this, for a single pin $(d \rightarrow \infty)$ the critical transverse field $H_{c 1}^{\perp}$, Eq.1.7 clearly vanishes in the thermodynamic $L \rightarrow \infty$ limit.

For a transverse field stronger than $H_{c 1}^{\perp}$ a continuous vortex lattice tilting transition takes place into a tilted (incommensurate) state. It proceeds via a proliferation of solitons, with soliton density $n_{s}\left(H_{\perp}\right)$ and the average vortex tilt at the location of the columnar defect growing continuously beyond $H_{c 1}^{\perp}$

$$
\begin{aligned}
n_{s}\left(H_{\perp}\right) & =\left.a^{-1}\left\langle\partial_{z} u\right\rangle\right|_{x=0}, \\
& \sim \begin{cases}0, & H<H_{c 1}^{\perp} \\
\left|H^{\perp}-H_{c 1}^{\perp}\right|, & H>H_{c 1}^{\perp},\end{cases}
\end{aligned}
$$

In the incommensurate phase, the screening length out to which the vortex lines are aligned along and pinned by the columnar defect diminishes with increasing soliton density and is given by

$$
\begin{aligned}
\lambda_{h} & =\left(\frac{B}{K}\right)^{1 / 2} \frac{1}{2 \pi n_{s}\left(H_{\perp}\right)}, \\
& \sim \frac{1}{\left|H^{\perp}-H_{c 1}^{\perp}\right|} .
\end{aligned}
$$

As we show below, for finite transverse field $H_{\perp}$, Friedel oscillations decay exponentially away from the columnar 
defect with length also given by $\lambda_{h}$. We estimate the upper-critical transverse field $H_{c 2}^{\perp}$, for which soliton lattice becomes dense, to be given by

$$
H_{c 2}^{\perp} \approx H_{c 1}^{\perp}+\frac{\phi_{0}}{2 \pi w \xi}
$$

In the presence of fluctuations, $H_{c 2}$ field marks a crossover from a nonlinear soliton tilted regime to a uniformly tilted state. For large $H_{\perp}$ exceeding $H_{c 2}^{\perp}$, the vortex lattice tilts smoothly and the screening length reduces to $\lambda_{h} \sim 1 / H^{\perp}$, in this limit coinciding with Friedel oscillations decay length found by Affleck, et $\mathrm{al}^{35}$.

The finite temperature CI phase boundary in Fig.3 is given by

$$
H_{c 1}^{\perp}(T) \approx H_{c 1}^{\perp}(0)\left(1-\frac{T}{T_{p}}\right)
$$

The corresponding upper-critical crossover field $H_{c 2}^{\perp}(T)$ (dashed curve in Fig.3) for weak pinning $\left(\xi_{0} \gg a \sqrt{K / B}\right)$ can be estimated by using $H_{c 1}(T)$ and $\xi(T)$ inside Eq.1.14,

$$
H_{c 2}^{\perp}(T) \approx H_{c 1}^{\perp}(T)+H_{c 2}^{\perp}(0)\left(\frac{a \sqrt{K / B}}{\xi_{0}}\right)^{T /\left|T_{p}-T\right|}
$$

For strong pinning $\left(\xi_{0} \ll a \sqrt{K / B}\right), H_{c 2}^{\perp}(T)$ is given by the above expression, but with $\xi_{0}$ set equal to $a \sqrt{K / B}$.

In the remainder of the paper we demonstrate results summarized above.

\section{MODEL}

\section{A. Vortex lattice elasticity with a transverse magnetic field}

In a type-II superconductor, for fields above a lowercritical field magnetic flux penetrates in a form of interacting vortex flux tubes, with average density determined by the applied magnetic field. ${ }^{1}$ At low temperature and in the absence of disorder, a periodic array (Abrikosov lattice) of repulsive, elastic vortex lines forms, whose elastic description ${ }^{49}$ can be derived from the Ginzburg-Landau theory for the superconducting order parameter, that itself, under certain conditions, is derivable from the microscopic theory of superconductivity. As usual, transcending such a detailed derivation, on sufficiently long length scale the elastic vortex lattice energy functional can be deduced purely on symmetry grounds. In $d$-dimensions, it is formulated in terms of a $d$-1-dimensional Eulerian phonon field (Goldstone mode of the spontaneously broken translational symmetry) $\mathbf{u}(\mathbf{x}, z)=\mathbf{x}-\mathbf{x}_{i}$ describing a transverse vortex lattice distortion at a $d$-dimensional position $(\mathbf{x}, z)$ relative to a perfect vortex array characterized by $\left(\mathbf{x}_{i}, z\right)$.
A planar vortex array, that we take to be confined to the $x-z$ plane, is characterized by a scalar phonon field $u(x, z)$, describing $x$-directed vortex distortion with a continuum elastic Hamiltonian given by

$$
\mathcal{H}_{e l}=\frac{1}{2} \int d x d z\left[K\left(\partial_{z} u-h\right)^{2}+B\left(\partial_{x} u\right)^{2}\right],
$$

where $K$ and $B$ are tilt and compressional elastic moduli, respectively, that we take to be phenomenological parameters. ${ }^{50}$ The parameter $h$ encodes the effect of an additional magnetic field $H_{\perp}$, applied transversely to the columnar defect $(z-)$ axis, with $h=H_{\perp} /\left(\phi_{0} n_{0}^{2}\right)=$ $H_{\perp} / H_{z}$ in a sample that (other than the pin) we take for simplicity to be isotropic. ${ }^{56}$

Vortex pinning, characterized by a weak potential $V_{\text {pin }}(x, z)$ can be easily incorporated through its coupling to the local vortex density $n_{v}(x, z)$ via

$$
\mathcal{H}_{p}=\int d x d z V_{\text {pin }}(x, z) n_{v}(x, z) .
$$

As with any periodic elastic medium, vortex density is given by

$$
n_{v}(x, z) \approx n_{0}-n_{0} \partial_{x} u+\sum_{G_{p}} n_{G_{p}} e^{i G_{p}(x+u(x, z))},
$$

with vortex lattice distortion $u(x, z)$ entering through the variation of the long-scale density fluctuation, $-n_{0} \partial_{x} u$, and via the variation of the phase, $G_{p} u$ of the vortex density wave given by the last term. In above, $G_{p}=2 \pi n_{0} p=2 \pi p / a$ ( $a$ the vortex lattice constant, $p \in Z$ ) spans a one-dimensional reciprocal (to $x$ ) lattice and $n_{0}=1 / a$ the average $x$-projected vortex $1 \mathrm{D}$ density. Above representation for $n_{v}(x, z)$ can be derived in a standard way from its microscopic definition $n_{v}(x, z)=\sum_{i} \delta\left(x-x_{i}(z)\right)$ in terms of vortex-line configurations $x_{i}(z)$, by the use of the Poisson summation formula $^{37}$, with the key periodic (last) term arising from vortex discreteness.

For the problem of a single $z$-directed columnar defect, we can approximate the pinning potential by an attractive zero-range form $V_{\text {pin }}=-V_{0} c \delta(x)$, with $V_{0}$ and $c$ its effective strength and range, respectively. For simplicity, and without loss of qualitative generality, we include only the lowest harmonic, characterized by the minimal reciprocal lattice constants $G_{ \pm 1} \equiv \pm G= \pm 2 \pi / a$ to model the periodic vortex density. Furthermore, by minimizing the total energy, it is easy to show that the long-range part of the density variation, $-n_{0} \partial_{x} u$ has a simple effect of a small shift in vortex positions, $u_{0}(x, z)=-\frac{V_{0} n_{G} c}{B} \operatorname{sgn}(x)$, that is constant and positive to the left of the defect and constant and negative to the right of the defect. It thereby slightly increases the average vortex density, but only at the location of the columnar defect, $x=0$, that can be absorbed into the background density. Dropping an unimportant constant, the pinning Hamiltonian then reduces to

$$
\mathcal{H}_{p}=-v \int d z \cos (G u(0, z))
$$


localized at the defect at $x=0$, with $v \equiv 2 n_{G} V_{0} c$. The resulting total Hamiltonian, $\mathcal{H}=\mathcal{H}_{e l}+\mathcal{H}_{p}$

$$
\begin{aligned}
\mathcal{H}= & \frac{1}{2} \int d x d z\left[K\left(\partial_{z} u-h\right)^{2}+B\left(\partial_{x} u\right)^{2}\right] \\
& -v \int d z \cos (G u(0, z)),
\end{aligned}
$$

is reminiscent of the well-known sine-Gordon model describing a broad spectrum of commensurability phenomena in condensed matter physics, ranging from crystal surface roughness to topological defects in ordered media $^{41-43}$

There is, however, an essential difference in that the nonlinear pinning term is localized at $x=0$. As a result, away from the defect, the system is harmonic and therefore solvable by elementary methods. Clearly, as illustrated in Fig. 2 away from the defect the vortex lattice must asymptote to that of a columnar defect-free configuration, that simply follows the transverse field

$$
u_{\infty}(x, z)=h z, \quad \text { for } x \rightarrow \pm \infty,
$$

obtained by minimizing $\mathcal{H}$ for $v=0$. As we will show below, we can fruitfully take advantage of the locality of the pinning potential by "integrating out" (eliminating) the bulk elastic degrees of freedom away from the defect, thereby reducing the two-dimensional problem to an effective one-dimensional nonlinear one, that can be solved exactly. The approach is quite similar to Kane-Fisher's treatment of a point impurity in a one-dimensional electron liquid. ${ }^{38}$

\section{B. Reduction to one-dimensional model}

As noted above, because of the short-range nature of the pinning potential in $\mathcal{H}$, Eq.2.5, vortex degrees of freedom, $u(x, z)$ away from the columnar defect at $x=0$, are governed by a harmonic Hamiltonian. As a result, they are simply related to vortex distortion at the columnar defect, allowing us to eliminate $u(x, z)$ in favor of $u(0, z)$. To automatically satisfy the boundary conditions $\left.\partial_{z} u(x, z)\right|_{z=0, L}=h$, induced by finite $h$, it is convenient to shift to a new phonon variable, measuring vortex lattice distortion relative to $u_{\infty}$,

$$
\tilde{u}(x, z)=u(x, z)-h z,
$$

in terms of which the Hamiltonian becomes

$$
\begin{aligned}
\mathcal{H}= & \frac{1}{2} \int d x d z\left[K\left(\partial_{z} \tilde{u}\right)^{2}+B\left(\partial_{x} \tilde{u}\right)^{2}\right] \\
& -v \int d z \cos [G(\tilde{u}(0, z)+h z)] .
\end{aligned}
$$

At zero temperature a reduction to an effectively onedimensional model can be most straightforwardly done by solving the Euler-Lagrange equation for $u(x, z)$ for a prescribed (but arbitrary) distortion $u(0, z) \equiv u_{0}(z)$ on the columnar defect (In Appendix A, we present a complementary constrained functional integral-based derivation of above result, that extends to finite temperature)

$$
\left(K \partial_{z}^{2}+B \partial_{x}^{2}\right) u(x, z)=B u_{0}(z) \partial_{x} \delta(x) .
$$

The local stress term (source term on the right-hand-side) is chosen so as to produce a vortex lattice distortion at $x=0$ to automatically satisfy the boundary condition $u(0, z) \equiv u_{0}(z)$. Standard Fourier analysis leads to a solution

$$
\tilde{u}\left(x, q_{z}\right)=\tilde{u}_{0}\left(q_{z}\right) e^{-(K / B)^{1 / 2}\left|q_{z}\right||x|},
$$

which, when substituted into the Hamiltonian, $\mathcal{H}$, Eq.2.8, and integrating over $x$ reduces to

$$
\begin{aligned}
\mathcal{H}_{0}= & \sqrt{K B} \int \frac{d q_{z}}{2 \pi}\left|q_{z}\right|\left|\tilde{u}_{0}\left(q_{z}\right)\right|^{2} \\
& -v \int d z \cos \left[G\left(\tilde{u}_{0}(z)+h z\right)\right] .
\end{aligned}
$$

The two-dimensional nature of the underlying vortex lattice is captured by the nonanalytic form $\left(\left|q_{z}\right|\right)$ of the effective one-dimensional elasticity in $\mathcal{H}_{0}$. As in other examples of a low-dimensional system coupled to a bulk system (e.g., a crack or a crystal surface in a bulk solid ${ }^{58}$ ) it encodes long-range interactions of one-dimensional deformations mediated through bulk (away from the columnar defect) degrees of freedom, as can be easily seen by reexpressing $\mathcal{H}_{0}$ in terms of $\tilde{u}_{0}(z)$

$$
\begin{aligned}
\mathcal{H}_{0}= & \frac{\sqrt{K B}}{2 \pi} \iint d z d z^{\prime}\left(\frac{\tilde{u}_{0}(z)-\tilde{u}_{0}\left(z^{\prime}\right)}{z-z^{\prime}}\right)^{2} \\
& -v \int d z \cos \left[G\left(\tilde{u}_{0}(z)+h z\right)\right] .
\end{aligned}
$$

The long-range elasticity qualitatively distinguishes this system from a one-dimensional sine-Gordon model characterized by short-range and therefore analytic $\left(q^{2}\right)$ long-scale elasticity. As we will see in Sec.IV, the associated enhanced stiffening of elastic distortions is what allows this one-dimensional system to undergo a finite temperature roughening phase transition, in contrast to a one-dimensional sine-Gordon model. For reasons that will become clear below and by the analogy with the sineGordon model, we refer to this system as the sine-Hilbert model. In the next section we will study the sine-Hilbert model at zero temperature but finite tilting field $h$, in order to characterize a vortex lattice response to a magnetic field $H_{\perp}$ applied transversely to the columnar defect.

\section{ZERO-TEMPERATURE TRANSVERSE FIELD RESPONSE}

\section{A. Bulk tilt response}

For $h=0$ much is known about the sine-Hilbert model, $\mathcal{H}_{0}$, Eqs.2.11,2.12, as it arises in many different 
physical contexts including resistively-shunted Josephson junctions ${ }^{44}$ and Luttinger liquid transport in the presence of an impurity ${ }^{38}$. In the present context, these findings relate to a vortex lattice at finite temperature, a study that we will undertake in Sec.IV. Furthermore, using inverse scattering transform Santini, Ablowitz and Fokas ${ }^{59}$ have shown that the classical sine-Hilbert model is integrable and admits soliton solutions, a finding that we will make use of below.

Less is known about the finite $h$ phenomenology, the study of which is facilitated by returning to the $u_{0}(z)=$ $\tilde{u}_{0}(z)+h z$ displacement field. In terms of $u_{0}(z)$, the Hamiltonian becomes:

$$
\begin{aligned}
\mathcal{H}_{0}= & \frac{\sqrt{K B}}{2 \pi} \iint d z d z^{\prime}\left(\frac{u_{0}(z)-u_{0}\left(z^{\prime}\right)-h\left(z-z^{\prime}\right)}{z-z^{\prime}}\right)^{2} \\
& -v \int d z \cos \left[G u_{0}\right] .
\end{aligned}
$$

For a finite transverse field, a good starting reference point are two competing solutions

$$
\begin{aligned}
& u_{C}(z)=0, \\
& u_{I}(z)=h z,
\end{aligned}
$$

that minimize $\mathcal{H}_{0}\left[u_{0}(z)\right]$ in the $h \rightarrow 0$ and $h \rightarrow \infty$ limits, respectively. By analogy with commensurateincommensurate (CI) phase transitions ${ }^{42,43}$ we refer to the corresponding phases as "commensurate" and "incommensurate", respectively. In the commensurate state, $u_{C}(z)$, vortex lattice aligns with the columnar defect, minimizing the pinning energy, while raising the diamagnetic energy. In the incommensurate state, $u_{I}(z)$, instead, the vortex lattice aligns with the external field, thereby ignoring the defect and sacrificing its attractive pinning energy. For a sample of extent $L$ along $z$, the corresponding total energies in the two cases are given by

$$
\begin{aligned}
E_{C} & =\frac{\sqrt{B K}}{2 \pi} h^{2} L^{2}-v L, \\
E_{I} & =0 .
\end{aligned}
$$

The extensive scaling, $\sim L^{2}$ of the magnetic energy in $E_{c}$ is expected from Eq.2.10, that shows that elastic distortion on scale $L$ along $z$, imposed at $x=0$ (in the commensurate phase corresponding to misalignment with applied field) decays over length $L(B / K)^{1 / 2}$ into the bulk, leading to a region of area $\sim L^{2}(B / K)^{1 / 2}$ with a finite diamagnetic energy cost. As discussed in the Introduction, in the thermodynamic limit this bulk diamagnetic energy always dominates over the linear-with $L$ pinning energy, and vortex system is in the incommensurate state for arbitrarily small transverse field $h$. However, for a finite $L$, we find from Eqs.3.4, 3.5, that for a single pin, the tilting transition between $u_{C}$ and $u_{I}$ states takes place at the critical ("thermodynamic bulk") tilting field

$$
h_{c}=\left(\frac{2 \pi v}{\sqrt{B K}}\right)^{1 / 2} \frac{1}{L^{1 / 2}},
$$

that, as expected, vanishes in the thermodynamic limit, $L \rightarrow \infty$. For a finite density of columnar defects spaced by $d$, it is clear that the bulk critical field saturates at a finite $d$-dependent value given by

$$
h_{c} \approx\left\{\begin{array}{ll}
\left(\frac{2 \pi v}{\sqrt{B K}}\right)^{1 / 2} \frac{1}{L^{1 / 2}}, & L \sqrt{\frac{B}{K}} \ll d \\
\left(\frac{2 \pi v}{K}\right)^{1 / 2} \frac{1}{d^{1 / 2}}, & L \sqrt{\frac{B}{K}} \gg d
\end{array},\right.
$$

This oversimplified picture of the tilting transition, ignoring solitons (see below) is summarized in Fig.4.

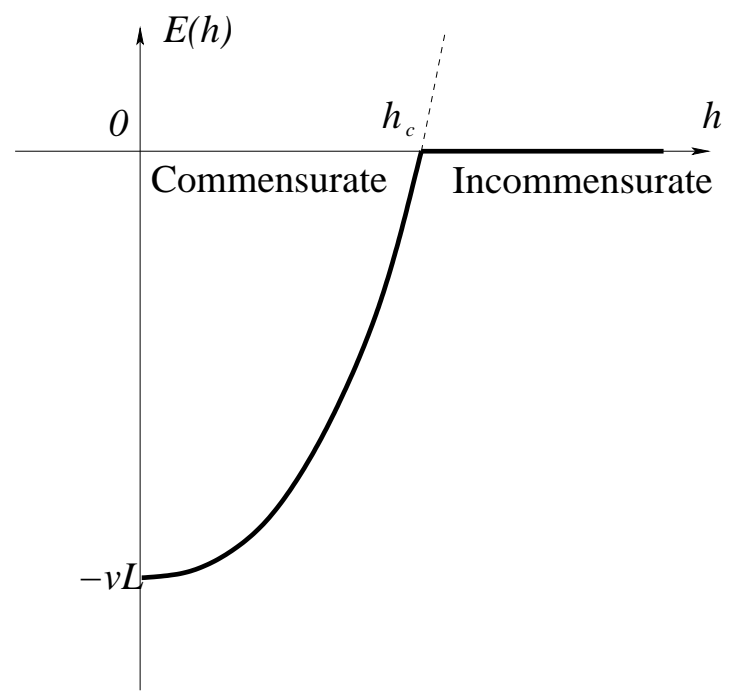

FIG. 4: Oversimplified (ignoring solitons) expression for the energy $E(h)$ of a vortex lattice in the presence of a transverse field $h$, indicating the aligned (C) to tilted (I) transition at $h_{c}$.

\section{B. Tilt-solitons}

Two states, $u_{C}, u_{I}$, are only appropriate in the $h \rightarrow 0$, $h \rightarrow \infty$ limits, respectively. However, in analogy with other systems, where there is competition between elastic and pinning energies ${ }^{41-43}$, we expect and find (see below), that the tilting transition into an incommensurate state is driven by soliton proliferation transition above a lower-critical field $h_{c 1}$, that preempts the bulk transition at $h_{c}$ found above. ${ }^{61}$ The soliton state above $h_{c 1}$ then continuously approaches the fully incommensurate $u_{I}(z)$ solution in the $h \rightarrow \infty$ limit, when solitons become dense. ${ }^{61}$ The existence of a lower-energy soliton solution can be seen by a simple inspection of the Hamiltonian $\mathcal{H}_{0}$, Eq.3.1. It stems from the periodicity of the pinning energy, $\mathcal{H}_{\text {pin }}\left[u_{0}\right]=\mathcal{H}_{\text {pin }}\left[u_{0}+a\right]$, that microscopically corresponds to its independence of which of the identical vortex lines in the array is pinned by the columnar defect. A soliton at $z_{0}$ corresponds to a solution $u_{0 s}(z)$ that switches at $z_{0}$ between two adjacent minima of the periodic potential. As illustrated in Fig.5, from the 2D per- 
spective such soliton describes a switching between two adjacent vortex lines localized on the columnar defect.

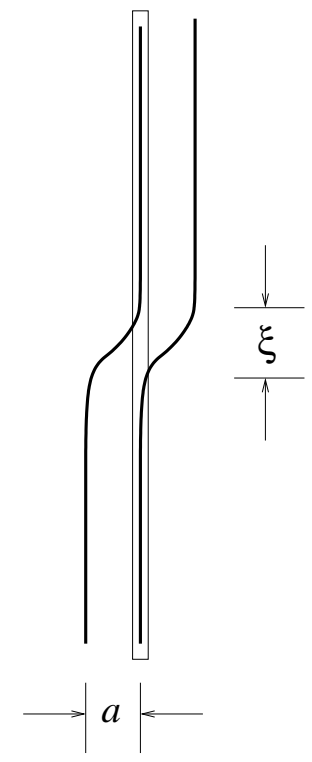

FIG. 5: A two-dimensional perspective of a 1D soliton along $z$, corresponding of an exchange between two neighboring vortex lines localized on the columnar defect.

At zero temperature the soliton shape is characterized by a single length scale,

$$
\xi_{0}=\left(\frac{a}{2 \pi}\right)^{2} \frac{2 \sqrt{B K}}{v}
$$

that can be read off from $\mathcal{H}_{0}$ by balancing the elastic and pinning energies, that scale like $\sqrt{K B} a^{2}$ and $v \xi_{0}$, respectively. This soliton width is set by the balance between the pinning and elastic energies. The former (latter) is minimized by the most (least) abrupt shift between the adjacent pinning minima, with $\xi_{0}$ reflecting this through its dependence on elastic moduli and pinning strength, increasing with $B, K$ and decreasing with $v$. We note that the effective $1 \mathrm{D}$ modulus is a geometrical mean of the vortex compressional (B) and tilt (K) moduli, as 2D distortion corresponding to the soliton configuration along the defect in Fig.5 involves both tilt and compression of the $2 \mathrm{D}$ vortex lattice.

It is convenient to express length scales along $z$ in units of the soliton width, $\xi_{0}$, and trade in the displacement field $u_{0}(z)$ for a dimensionless phase field

$$
\phi(\hat{z})=\frac{2 \pi}{a} u_{0}(z),
$$

where throughout the paper we will used "hat'ed" symbols to denote dimensionless quantities. The Hamiltonian $\mathcal{H}_{0}$ then reduces to a dimensionless form

$$
\begin{aligned}
\frac{\mathcal{H}_{0}}{\epsilon_{0}}= & \frac{1}{4 \pi} \iint d \hat{z} d \hat{z}^{\prime}\left(\frac{\phi(\hat{z})-\phi\left(\hat{z}^{\prime}\right)-\hat{h}\left(\hat{z}-\hat{z}^{\prime}\right)}{\hat{z}-\hat{z}^{\prime}}\right)^{2} \\
& -\int d \hat{z} \cos [\phi],
\end{aligned}
$$

where

$$
\begin{aligned}
\epsilon_{0} & =\left(\frac{a}{2 \pi}\right)^{2} 2 \sqrt{B K}, \\
& =\xi_{0} v,
\end{aligned}
$$

is the soliton energy scale and

$$
\hat{h} \equiv \frac{2 \pi \xi_{0}}{a} h
$$

is a dimensionless measure of a transverse field.

These energy and length scales are sufficient to determine all qualitative ingredients of the soliton-driven tilting (CI) transition. However, integrability of the EulerLagrange equation $\frac{\delta \mathcal{H}_{0}}{\delta u_{0}(z)}=0$

$$
\frac{1}{\pi} \int d \hat{z}^{\prime} \frac{\phi(\hat{z})-\phi\left(\hat{z}^{\prime}\right)}{\left(\hat{z}-\hat{z}^{\prime}\right)^{2}}+\sin \phi(\hat{z})=0,
$$

found by Santini, et al. ${ }^{59}$ allows for a quantitatively exact analysis. In above, the integral must be defined as the principle value with the singular point $z=z^{\prime}$ excluded, and we have used free boundary conditions on $\phi(L)$ and $\phi(0)$ in deriving Eq.3.14. These lead to a boundary condition

$$
\left.\partial_{\hat{z}} \phi(\hat{z})\right|_{\hat{z}=0, \hat{L}}=\hat{h},
$$

that supplements Eq.3.14. It encodes the condition that at the edge of the sample vortex lines tilt to slope $h$ to follow the external magnetic field.

A single-soliton solution to the above sine-Hilbert equation, Eq.3.14 was discovered by Rudolf Peierls ${ }^{60}$ in his seminal study of an edge dislocation in a crystal, and later rediscovered and considerably extended (to multisolitons, dynamics and proving integrability) using the inverse scattering method by Santini, et al. ${ }^{59}$. It is illustrated in Fig.6 and given by

$$
\phi_{s}(\hat{z})=-2 \operatorname{ArcTan} \frac{1}{\hat{z}-\hat{z}_{0}},
$$

This solution can be verified by a direct substitution of $\phi_{s}(z)$ into Eq.3.14, using Hilbert transforms that we review in Appendix D.

Substituting $\phi_{s}(\hat{z})$ into $\mathcal{H}_{0}$, Eq.3.10, and using Hilbert transforms (with details presented in Appendix E), we find the energy of a single soliton state

$$
\begin{aligned}
E_{1} & =\mathcal{H}_{0}\left[\phi_{s}(\hat{z})\right], \\
& \equiv \epsilon_{0} \hat{E}_{C}+\epsilon_{0} \hat{E}_{s 1}
\end{aligned}
$$

where dimensionless single soliton energy, $\hat{E}_{s 1}$, computed in Appendix E is given by

$$
\hat{E}_{s 1}=2 \pi \ln \left(\frac{e \hat{L}}{4}\right)-\hat{h}(2 \hat{L}-2 \pi),
$$

$\hat{L} \equiv L / \xi_{0}$, and $\hat{E}_{C}$ the dimensionless energy of the commensurate state (cf. Eq.3.4)

$$
\hat{E}_{C}=\frac{1}{4 \pi} \hat{h}^{2} \hat{L}^{2}-\hat{L}
$$




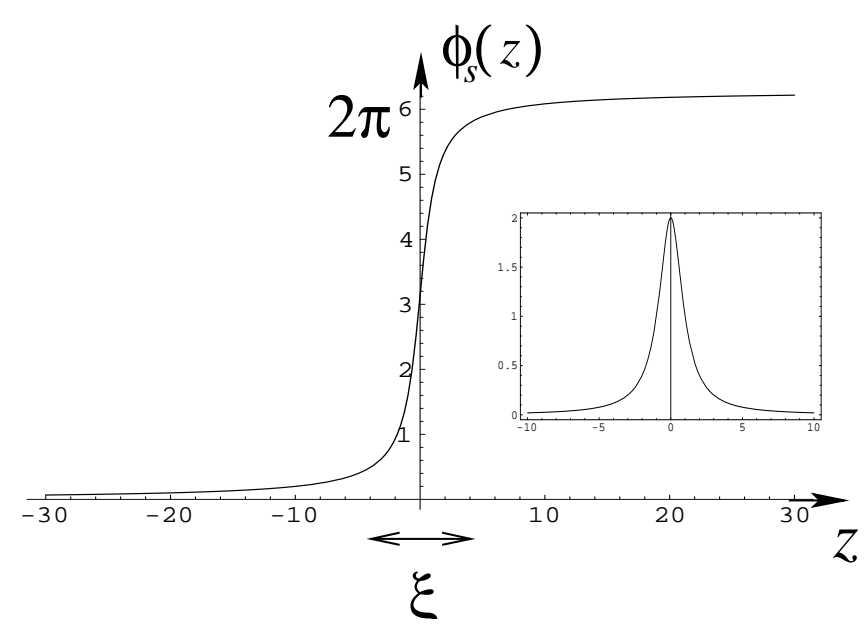

FIG. 6: Soliton solution $\phi_{s}(z)$, Eq.3.16 to the sine-Hilbert equation, Eq.3.14. Inset shows $\partial_{z} \phi_{s}(z)$.

From $\hat{E}_{s 1}$, Eq.3.19 it is clear that soliton energy becomes negative for $\hat{h}>\hat{h}_{c 1}$, with

$$
\begin{aligned}
h_{c 1} & =\frac{a}{\xi_{0}} \frac{1}{2 \hat{L}-2 \pi} \ln \frac{e \hat{L}}{2}, \\
& \approx \frac{a}{2 L} \ln \frac{L}{\xi_{0}},
\end{aligned}
$$

that, as expected (given that there is only a single pin) vanishes in the thermodynamic limit. As with other CI phase transitions ${ }^{42,43}$, this leads to soliton proliferation at $h_{c 1}{ }^{61}$, that preempts the bulk transition at $h_{c}$, approaching the fully tilted incommensurate state $u_{I}(z)$, only as $h \rightarrow h_{c 2}$. The latter tilt field is defined by when the solitons begin to overlap, namely $n_{s}\left(h_{c 2}\right) \approx \xi_{0}^{-1}$. However, in contrast to conventional CI transition, where the ratio of the lower critical field to the thermodynamic one is of order 1 constant $(=4 /(\sqrt{2} \pi) \approx 0.90$ for the CI transition in the sine-Gordon model), here

$$
\frac{h_{c 1}}{h_{c}}=\frac{\pi^{1 / 2}}{2}\left(\frac{\xi_{0}}{L}\right)^{1 / 2} \ln \frac{L}{\xi_{0}} .
$$

Vanishing of this ratio for large $L$ demonstrates the importance of solitons in driving the vortex lattice tilting transitions.

\section{Tilt-soliton proliferation transition}

The vortex lattice tilting angle, $\theta(h)$, related to transverse flux density $B_{\perp}\left(H_{\perp}\right)$, via $b_{\perp} \equiv \tan \theta=B_{\perp} / B_{z}$ is determined by the soliton density $n_{s}(h)$ through the relation

$$
b_{\perp}(h)=a n_{s}(h)
$$

The soliton density $n_{s}(h)$ for $h>h_{c 1}$ in turn is determined by the balance of the soliton chemical potential en$\operatorname{ergy} h$ (that induces solitons) and the soliton repulsive interaction. In the dilute soliton limit (for $h_{c 1}<h \ll h_{c 2}$ ), we can approximate the latter by a sum of pair-wise soliton interactions. This is determined by the energy $E_{2}\left(\hat{z}_{1}, \hat{z}_{2}\right) \equiv \epsilon_{0} \hat{E}_{2}\left(\hat{z}_{1}, \hat{z}_{2}\right)$ of two one-solitons, localized at $\hat{z}_{1}$ and $\hat{z}_{2}$, separated by a large distance $\hat{z}_{1}-\hat{z}_{2} \gg 1$, with

$$
\begin{aligned}
\hat{E}_{2}\left(\hat{z}_{1}, \hat{z}_{2}\right) & \equiv \hat{\mathcal{H}}_{0}\left[\phi_{s 1}+\phi_{s 2}\right] \\
& =\frac{1}{4 \pi} \iint d \hat{z} d \hat{z}^{\prime}\left(\frac{\phi_{s 1}(\hat{z})+\phi_{s 2}(\hat{z})-\phi_{s 1}\left(\hat{z}^{\prime}\right)-\phi_{s 2}\left(\hat{z}^{\prime}\right)-\hat{h}\left(\hat{z}-\hat{z}^{\prime}\right)}{\hat{z}-\hat{z}^{\prime}}\right)^{2}-\int d \hat{z} \cos \left[\phi_{s 1}(\hat{z})+\phi_{s 2}(\hat{z})\right]
\end{aligned}
$$

A straightforward calculation along the lines of the computation of $E_{1}$ gives (with details presented in Appendix F):

$$
\hat{E}_{2}\left(\hat{z}_{1}, \hat{z}_{2}\right)=\hat{E}_{C}+2 \hat{E}_{s 1}(\hat{h})+V_{s}\left(\hat{z}_{1}-\hat{z}_{2}\right),
$$

where the soliton two-body repulsive interaction is given by

$$
\begin{aligned}
V_{s}(\hat{z}) & =2 \pi \ln \left[\frac{(\hat{L} / 2)^{2}}{\hat{z}^{2}+4}\right]+4 \pi \\
& \approx 4 \pi \ln \frac{\hat{L}}{2|\hat{z}|}+4 \pi, \text { for } 1 \ll|\hat{z}| \ll \hat{L} / 2,
\end{aligned}
$$

and is illustrated in Fig.7.
The energy of the $N_{s}$ soliton lattice is then given by

$$
\begin{aligned}
\hat{E}_{N s} & =\hat{E}_{C}+N_{s} \hat{E}_{s 1}+\sum_{i<j} V_{s}\left(\hat{z}_{i}-\hat{z}_{j}\right), \\
& \approx \hat{E}_{C}+2 \hat{L}\left(\hat{h}_{c 1}-\hat{h}\right) N_{s}+\frac{1}{2} V_{s}(\hat{L} / 2) N_{s}^{2}, \\
& \approx \hat{E}_{C}+\hat{L}^{2}\left[2\left(\hat{h}_{c 1}-\hat{h}\right) \hat{n}_{s}+\frac{1}{2} V_{s}(\hat{L} / 2) \hat{n}_{s}^{2}\right],
\end{aligned}
$$

where, because of the long-range nature (logarithmic) of the interaction $V_{s}(\hat{z})$, the sum in the interaction energy is dominated by the longest length scale, $L$, leading to its $N_{s}^{2}=n_{s}^{2} L^{2}$ stronger-than-extensive growth. This is in qualitative contrast to standard CI $T=0$ 


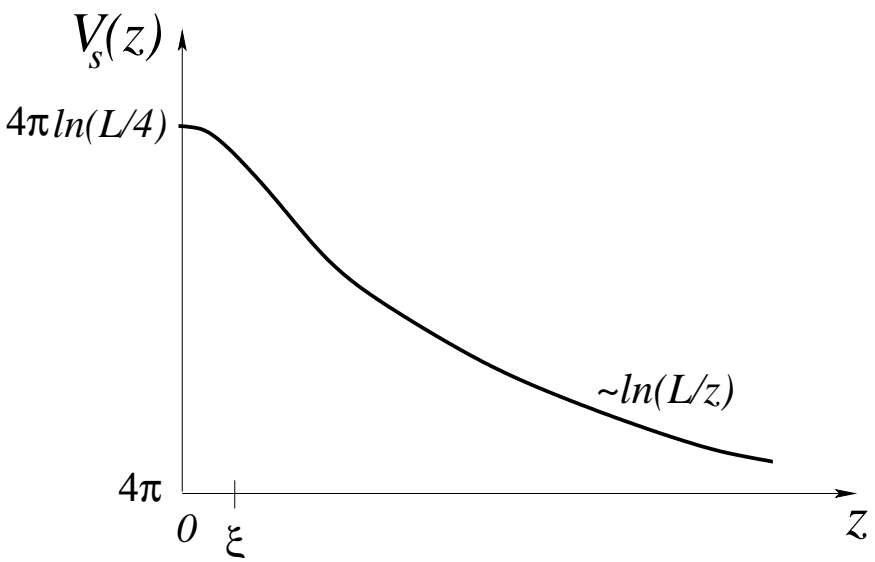

FIG. 7: Soliton interaction potential $V_{s}(z)$, illustrating a longrange logarithmic repulsion.

transitions ${ }^{42,43,61}$, where the interaction is short-ranged and the corresponding sum is dominated by the smallest term $V_{s}\left(a / \xi_{0}\right)$, leading to an extensive interaction energy. ${ }^{62}$

Minimizing $\hat{E}_{N s}\left[\hat{n}_{s}\right]$ over the soliton density $\hat{n}_{s}=$ $N_{s} / \hat{L}$, we find the advertised CI soliton proliferation transition $^{48}$ at $\hat{h}_{c 1}$, with the soliton density

$$
\hat{n}_{s}(\hat{h})= \begin{cases}0, & \hat{h}<\hat{h}_{c 1} \\ \hat{n}_{s 0}\left(\hat{h}-\hat{h}_{c 1}\right), & \hat{h}>\hat{h}_{c 1},\end{cases}
$$

growing linearly with the transverse field $h . \hat{n}_{s 0}$ is $O(1)$ constant given by

$$
\begin{aligned}
\hat{n}_{s 0} & \equiv \frac{2}{V_{s}(\hat{L} / 2)}, \\
& \approx \frac{1}{2 \pi},
\end{aligned}
$$

The final numerical result, Eq.3.35, above is quite crude, providing only an order of magnitude estimate, as we have not carefully treated the case of a soliton near the edge of the system.

The dimensionless soliton density $\hat{n}_{s}$ (measured in units of $\left.1 / \xi_{0}\right)$ increases to $O(1)$ when $\hat{h}=h \frac{2 \pi \xi_{0}}{a}$ exceeds $\hat{h}_{c 1}$ by 1 . This corresponds to the physical soliton density $n_{s}\left(h_{c 2}\right) \approx 1 / \xi_{0}$ of a dense lattice of overlapping solitons, with the upper-critical transverse field $h_{c 2}$ given by

$$
h_{c 2}=h_{c 1}+\frac{a}{2 \pi \xi_{0}},
$$

consistent with physical picture illustrated in Fig.16 and summarized by Fig.8

Using solution $N_{s}(\hat{h})=\hat{n}_{s}(\hat{h}) \hat{L}$, Eq.3.33 inside $\hat{E}_{N s}$ we find the vortex lattice energy as a function of the transverse field $h$ :

$$
\hat{E}(\hat{h})= \begin{cases}\frac{\hat{h}^{2} \hat{L}^{2}}{4 \pi}-\hat{L}, & \hat{h}<\hat{h}_{c 1} \\ \frac{\hat{h}^{2} \hat{L}^{2}}{4 \pi}-\hat{L}-\frac{\hat{n}_{s 0}}{2}\left(\hat{h}-\hat{h}_{c 1}\right)^{2} \hat{L}^{2}, & \hat{h}_{c 1}<\hat{h} \ll \hat{h}_{c 2} .\end{cases}
$$

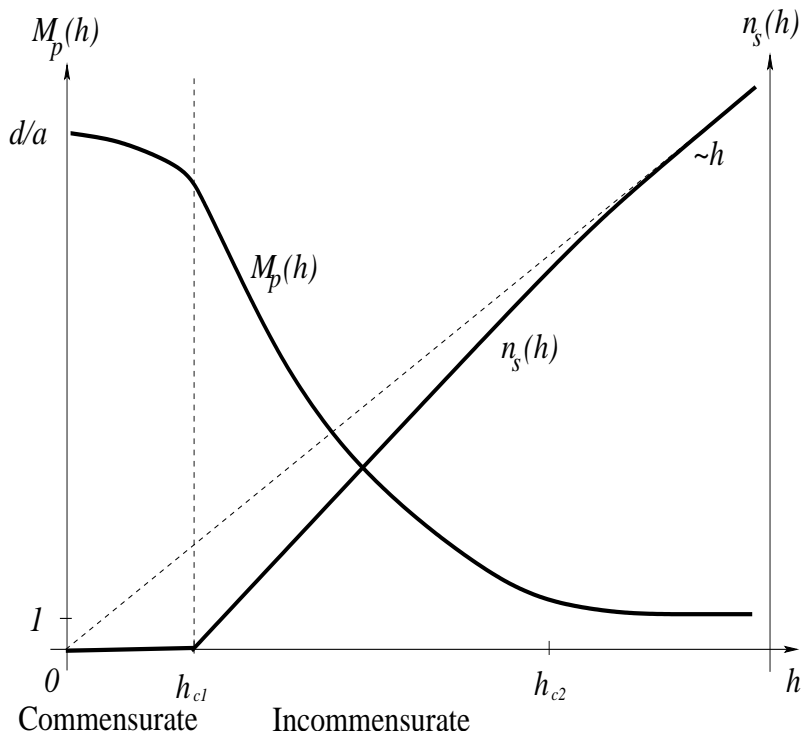

FIG. 8: A sketch of soliton density, $n_{s}(h)$, and the number of vortex lines pinned per columnar defect, $M_{p}(h)$, as functions of the transverse field $h$.

As expected the negative soliton energy (last term) cancels off the $\hat{L}^{2}$ misalignment-field energy (first term), as the incommensurate state is approached with increasing soliton density in the large $\hat{h} \gg \hat{h}_{c 1}$ limit. ${ }^{63}$ This energy and the corresponding phase diagram are illustrated in Fig.9.

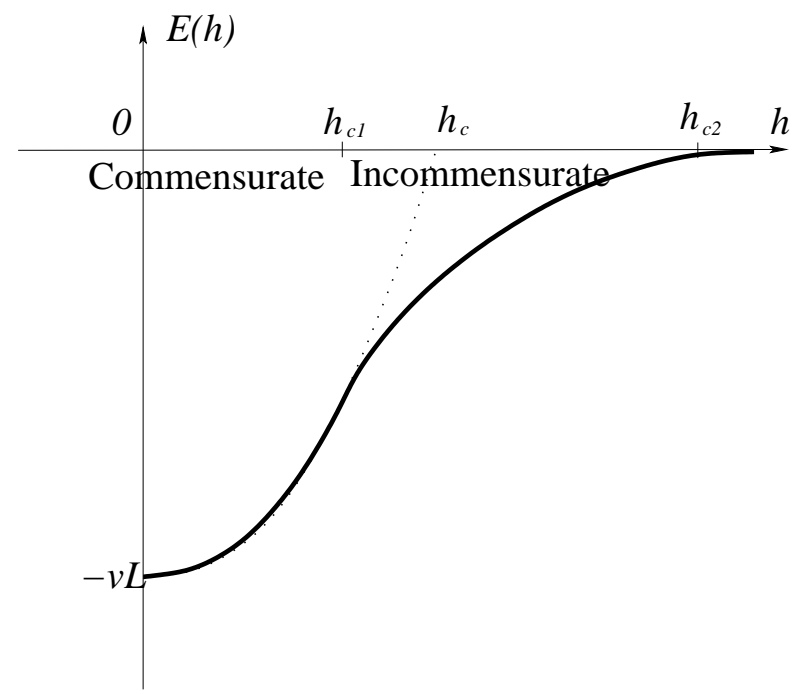

FIG. 9: Vortex lattice energy $E(h)$ as a function of transverse field $h$, illustrating soliton-driven tilting CI transition at the lower-critical field $h_{c 1}$ preempting the bulk thermodynamic critical field $h_{c}$. At high upper-critical transverse field $h_{c 2}$ the system crosses over to a fully discommensurated tilted state with a smooth tilt response. 


\section{Bulk vortex lattice distortions}

Having established the existence and computed the details of the $T=0$ vortex array tilting transition in terms of the one-dimensional field $u_{0}(z)$, illustrated in Fig.10, we now turn to the computation of the associated bulk vortex lattice distortion $u_{0}(x, z)$. The connection be-

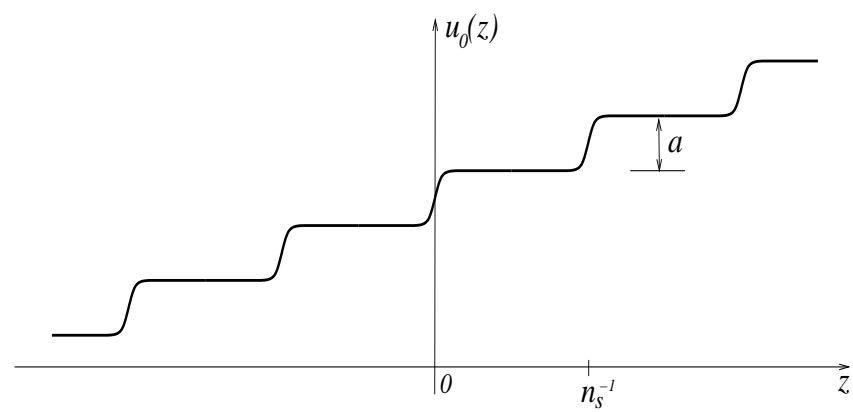

FIG. 10: A sketch of the function $u_{0}(z)$ for an array of 5 solitons.

tween $u_{0}(z)$ and $u_{0}(x, z)$ is established through a key relation, Eq.2.10 (here we use the subscript 0 to denote $T=0$ field configurations, obtained by minimizing the energy)

$$
\tilde{u}_{0}\left(x, q_{z}\right)=\tilde{u}_{0}\left(q_{z}\right) e^{-|x| / \lambda_{h}\left(q_{z}\right)}
$$

that shows quite clearly that a one-dimensional distortion with a wavevector $q_{z}$ along the defect penetrates into the bulk $x \neq 0$ over a screening length

$$
\lambda_{h}\left(q_{z}\right)=\left(\frac{B}{K}\right)^{1 / 2} \frac{1}{\left|q_{z}\right|} .
$$

A real-space one-dimensional distortion in an $N_{s}$ soliton state is given by

$$
\begin{aligned}
\tilde{u}_{0}(z) & =\sum_{\alpha=1}^{N_{s}} u_{s}\left(z-z_{\alpha}\right)-h z \\
& \equiv \bar{u}_{0}(z)-\left(h-n_{s} a\right) z
\end{aligned}
$$

with $u_{s}(z)=(a / 2 \pi) \phi_{s}(z)$ the single soliton solution from Eq.3.16. Physically $\partial_{z} \tilde{u}_{0}(z)$ is proportional to transverse magnetization. In Eq.3.41 we defined a periodic part coming from the soliton array (illustrated in Fig.11)

$$
\bar{u}_{0}(z)=\sum_{\alpha=1}^{N_{s}} u_{s}\left(z-z_{\alpha}\right)-n_{s} a z
$$

that oscillates around 0 with period $1 / n_{s}$, and the remaining misalignment part $h_{\mathrm{eff}} z$, with an effective tiltfield

$$
\begin{aligned}
h_{\mathrm{eff}} & =h-n_{s}(h) a, \\
& =h-2 \pi \hat{n}_{s 0}\left|h-h_{c 1}\right| .
\end{aligned}
$$

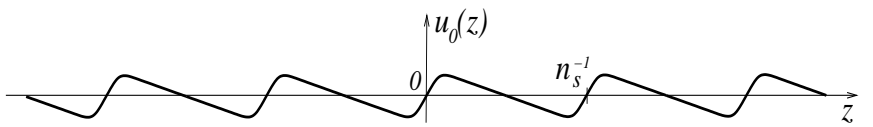

FIG. 11: A sketch of the function $\bar{u}_{0}(z)$, Eq.3.42, characterizing the periodic part of the soliton train solution $u_{0}$.

These respectively contribute to the oscillatory and uniform part of the (negative) transverse magnetization.

To compute the Fourier transform $\tilde{u}_{0}\left(q_{z}\right)$, we extended $h_{\text {eff }} z$ part of $\tilde{u}_{0}(z)$ beyond the system size $L$ to a continuous periodic function with period $2 L$ illustrated in Fig. 12 .

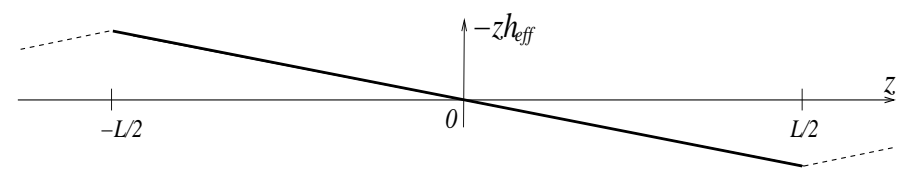

FIG. 12: A sketch of a tilted component of $u_{0}(z)$, periodically extended beyond the system size $L$.

From this decomposition it is clear that the distortion $\tilde{u}_{0}(z)$ appears on two characteristic wavelengths: the system size $L$ along $z$ (we take the system to be infinite along $x$ ) and the soliton spacing $n_{s}^{-1}$. Hence we predict that the associated bulk distortion, $u_{0}(x, z)$ will display an average misalignment with the applied field (i.e., on average align with the columnar defect), that extends over a length

$$
\lambda_{h}^{0}=\left(\frac{B}{K}\right)^{1 / 2} \frac{L}{\pi}
$$

along $x$ and will exhibit soliton-induced oscillations with wavelength $\sim n_{s}^{-1}$ that penetrate over scale

$$
\lambda_{h}=\left(\frac{B}{K}\right)^{1 / 2} \frac{1}{2 \pi n_{s}(h)},
$$

into the bulk, $x \neq 0$ away from the columnar defect.

Above qualitative discussion can be elevated to an exact calculation. Using Eq.3.38, the real-space bulk distortion is given by

$$
u_{0}(x, z)=h z+\int \frac{d q_{z}}{2 \pi} \tilde{u}_{0}\left(q_{z}\right) e^{-\left(\frac{K}{B}\right)^{\frac{1}{2}}\left|q_{z}\right||x|} e^{i q_{z} z}
$$

Putting together above ingredients, the Fourier transform $\tilde{u}_{0}\left(q_{z}\right)$ can be easily computed, and, when inserted into Eq.3.47 gives 


$$
\begin{aligned}
u_{0}(x, z) & =h z-\frac{4}{\pi^{2}} L h_{\mathrm{eff}} \sum_{m=0}^{\infty} \frac{(-1)^{m}}{(2 m+1)^{2}} e^{-\sqrt{\frac{K}{B}} \frac{\pi}{L}(2 m+1)|x|} \sin \left[\frac{\pi}{L}(2 m+1) z\right]+\frac{a}{\pi} \sum_{p=1}^{\infty} \frac{1}{p} e^{-\sqrt{\frac{K}{B}} 2 \pi n_{s} p|x|} \sin \left[2 \pi n_{s} p z\right] \\
& \approx\left[1-\left(1-\frac{n_{s}(h) a}{h}\right) e^{-\sqrt{\frac{K}{B}} \frac{\pi}{L}|x|}\right] h z+\frac{a}{\pi} e^{-\sqrt{\frac{K}{B}} 2 \pi n_{s}|x|} \sin \left[2 \pi n_{s} z\right]
\end{aligned}
$$

Although no approximation is required to compute $u_{0}(x, z)$, Eq.3.47, in Eq.3.48 we have considerably simplified the Fourier transform by approximating the soliton part $\bar{u}_{0}(z)$ by a function $-n_{s} a z$, periodically extended with period $n_{s}^{-1}$. This approximation is valid away from the dense soliton limit, i.e., for $h \ll h_{c 2}$, where $n_{s} a \ll 1$. In going to Eq.3.49 we have furthermore simplified $u_{0}(x, z)$ by keeping only the first harmonic in each of the two terms and approximated the first sine by a line with a proper slope (determined by boundary conditions) valid for $z \ll L$ away from the edges of the sample. The corresponding configurations of the vortex lattice in the commensurate state, $h<h_{c 1}$, slightly inside the soliton state, $h \approx h_{c 1}^{+}$, intermediate regime $h_{c 1}<h \ll h_{c 2}$, and deep in the soliton state $h \gg h_{c 1}$ are illustrated in Figs.13, 14, 15, and 16 respectively.

As anticipated by the qualitative discussion above, influence of the columnar defect depends on the tilt field $h$ and ranges from the maximum value $\lambda_{h}^{0}$, Eq.3.45 in the commensurate state, down to $\lambda_{h}$, Eq.3.46 in the soliton state. Upon increasing $h$ beyond the lower-critical field $h_{c 1}$, the soliton density increases and the penetration length decreases according to

$$
\begin{aligned}
\lambda_{h}(h) & =\frac{a}{4 \pi^{2} \hat{n}_{s 0}}\left(\frac{B}{K}\right)^{1 / 2} \frac{1}{\left|h-h_{c 1}\right|}, \\
& \rightarrow \lambda_{h}^{\infty} \equiv \frac{a}{2 \pi}\left(\frac{B}{K}\right)^{1 / 2} \frac{1}{h}, \text { for } h_{c 1} \ll h \ll h_{c 2},
\end{aligned}
$$

saturating at the microscopic lattice scale $a$ as $h_{c 2}$ is approached. The size of the pinned vortex "cloud", $\lambda_{h}$ allows to define an important dimensionless number $M_{p}(h)^{35,64}$

$$
M_{p}(h)=\frac{\lambda_{h}}{a},
$$

that gives the number of vortex lines effectively pinned by a single columnar defect. As illustrated in Fig. 8 it ranges from $M_{p}^{\max } \approx(B / K)^{1 / 2} L / a(d / a$ for finite density of pins) in the commensurate $\left(h<h_{c 1}\right)$ state down to its minimum value of 1 for $h \rightarrow h_{c 2}$.

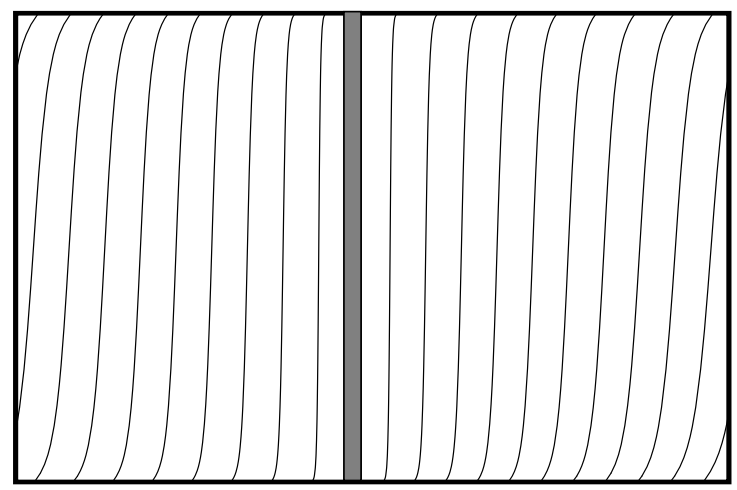

FIG. 13: Vortex lattice $(T=0)$ configuration $u_{0}(x, z)$ for a subcritical tilt field $h<h_{c 1}$, showing expulsion of tilt over a region of size $L \times(B / K)^{1 / 2} L$ around the pin, but with penetration of tilt on scale $\xi_{0}$ at $x=0$ growing with $|x|$ to full penetration beyond $|x| \approx(B / K)^{1 / 2} L$.

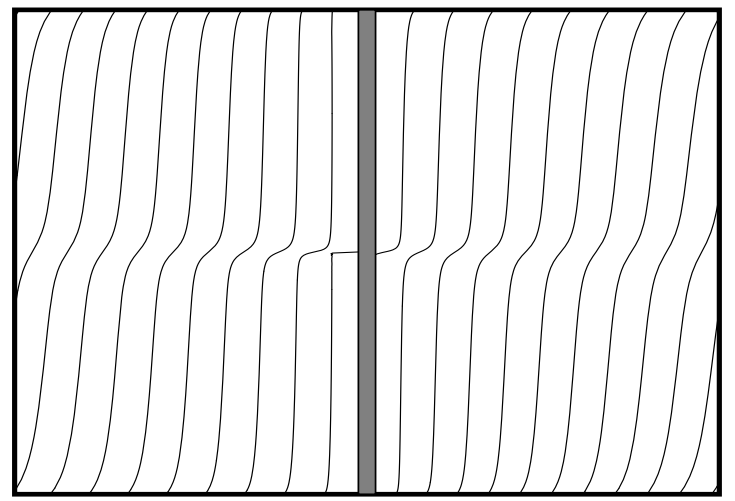

FIG. 14: Vortex lattice $(T=0)$ configuration $u_{0}(x, z)$ for justabove critical tilt-field $h=h_{c 1}^{+}$, with a single soliton along the pin. It shows the expulsion of tilt over a region $L \times$ $(B / K)^{1 / 2} L / 2$ around the pin, with the tilt penetration length $\lambda_{h 2}=(B / K)^{1 / 2} L / 2$ along $|x|$ reduced by a factor of 2 relative to the case of no solitons, illustrated in Fig.13.

\section{FINITE TEMPERATURE THERMODYNAMICS AND CORRELATIONS}

Having established the vortex lattice $T=0$ tiltresponse to a transverse magnetic field, we now turn to the study of finite-temperature properties of the vortex lattice pinned by a single columnar defect. ${ }^{35}$ As 


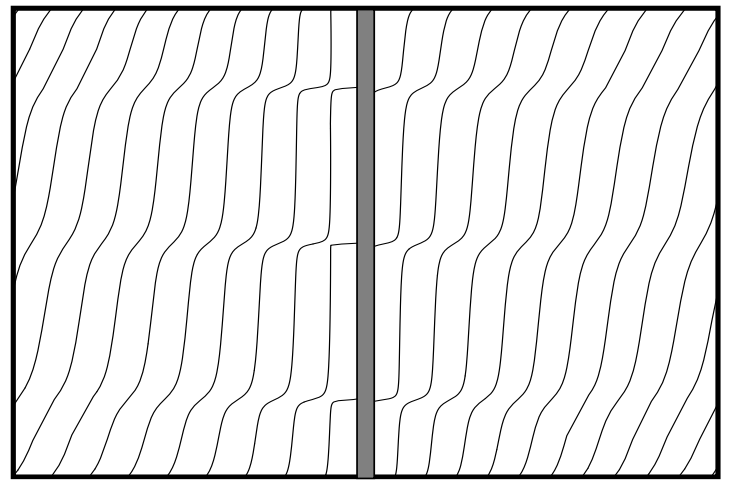

FIG. 15: Vortex lattice $(T=0)$ configuration $u_{0}(x, z)$ for tilt-field $h_{c 1}<h \ll h_{c 2}$, with three solitons along the pin. It shows the expulsion of tilt over a region $L \times(B / K)^{1 / 2} L / 4$ around the pin, with the tilt penetration length $\lambda_{h 4}=$ $(B / K)^{1 / 2} L / 4$ along $|x|$ reduced by a factor of 4 relative to the case of no solitons, illustrated in Fig.13. As discussed in the text, in general, the $N$-soliton penetration length is given by $\lambda_{h N}=\left(\frac{B}{K}\right)^{1 / 2} L / N_{s} \approx \lambda_{h} / N_{s}=n_{s}^{-1}(B / K)^{1 / 2}$.

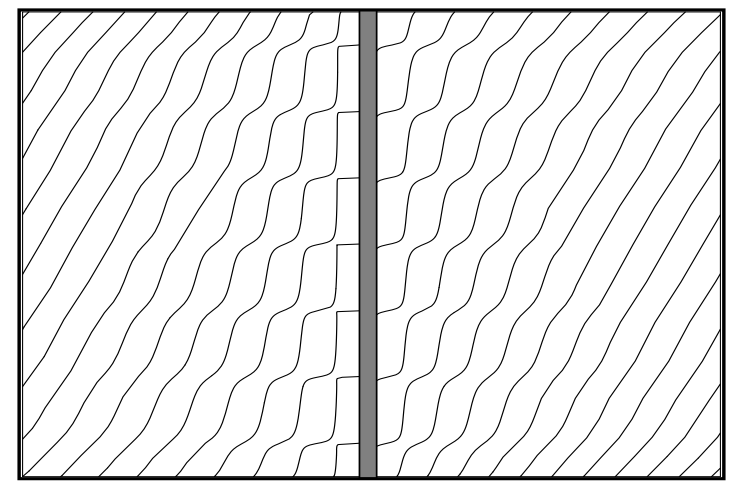

FIG. 16: Vortex lattice $(T=0)$ configuration $u_{0}(x, z)$ for tiltfield $h \rightarrow h_{c 2}$, giving a dense soliton array and a vanishing tilt-expulsion length. A resulting vortex lattice tilt response is nearly that of a pin-free system, with the distortion confined to the immediate vicinity of the columnar defect.

usual, the thermodynamics and corresponding correlation functions can be computed by integrating the vortex phonon configurations weighted by a Boltzmann factor, $e^{-\mathcal{H} / k_{B} T} / Z$, with $Z$ the corresponding partition function $Z=\int[d u] e^{-\mathcal{H} / k_{B} T}$.

\section{A. Thermal depinning transition for vanishing tilt-field, $h=0$}

We first consider the case of a vanishing tilt-field, $h=0$, and show that the effective one-dimensional, longrange interacting Hamiltonian, $\mathcal{H}_{0}$, to which our $2 \mathrm{D}$ vortex problem has been reduced, exhibits a thermal depinning transition, qualitatively similar to a well-known roughening transition in $2 \mathrm{D}$ systems. ${ }^{41-43}$
The indication of the existence of the transition comes from a computation of the thermodynamics perturbatively in powers of the pinning potential strength $v$. As for the $2 \mathrm{D}$ roughening (and related) transition, this perturbation theory diverges (at long length scales) for low temperatures $T<T_{p}$ even for an arbitrarily small $v$, but is convergent for high temperatures $T>T_{p}$, with the critical pinning temperature $T_{p}$ defined below. As usual to make sense of the associated infrared divergences near and below $T_{p}$ we employ the momentum-shell renormalization-group (RG) transformation ${ }^{65}$ on the $1 \mathrm{D}$ Hamiltonian $\mathcal{H}_{0}$

$$
\mathcal{H}_{0}=\sqrt{K B} \int \frac{d q_{z}}{2 \pi}\left|q_{z}\right|\left|\tilde{u}_{0}\left(q_{z}\right)\right|^{2}-v \int d z \cos \left[G \tilde{u}_{0}(z)\right] .
$$

Namely, in the partition function for the model, we integrate out perturbatively in $v$ short-scale phonon modes $u_{0}^{>}\left(q_{z}\right)$ in a narrow shell $G_{z} e^{-\delta \ell}<\left|q_{z}\right|<G_{z}$ around the short scale cutoff $G_{z}=G \sqrt{B / K} \equiv\left(2 \pi / a_{z}\right)$, with $\delta \ell \ll 1$. This allows us to express the partition function in terms of integrals over the remaining effective coursegrained phonon modes $u_{0}^{<}\left(q_{z}\right)$, with $\left|q_{z}\right|<G_{z} e^{-\delta \ell}$. In order to massage the resulting Hamiltonian into the original form $\mathcal{H}_{0}$, i.e., to bring the reduced ultra-violet (UV) cutoff back up to $G_{z}$, we rescale lengths and wavevectors according to

$$
\begin{aligned}
q_{z} & =q_{z}^{\prime} e^{-\delta \ell} \\
z & =z^{\prime} e^{\delta \ell}
\end{aligned}
$$

For convenience, to keep the vortex lattice reciprocal vector $G$ in the argument of the pinning potential fixed, we choose not to rescale the real-space phonon field $u_{0}^{<}(z)=u_{0}^{\prime}\left(z^{\prime}\right)$, which implies a rescaling

$$
u_{0}^{<}(q)=e^{-\delta \ell} u_{0}^{\prime}\left(q^{\prime}\right)
$$

for its Fourier transform. With these transformations the effective coarse-grained Hamiltonian returns to its original $\mathcal{H}_{0}$ form, but with effective, $\ell$-dependent elastic and pinning parameters. As can be easily checked, to lowest order in $v$, the one-dimensional stiffness $\sqrt{B K}$ remains unchanged, ${ }^{66}$ and the effective pinning strength transforms according to

$$
\begin{aligned}
v(\delta \ell) & =v e^{\delta \ell}\left\langle e^{i G u_{0}^{>}(z)}\right\rangle_{0}, \\
& =v e^{\delta \ell} e^{-\frac{1}{2} G^{2}\left\langle\left(u_{0}^{>}(z)\right)^{2}\right\rangle_{0}} \\
& \equiv v e^{(1-\eta / 2) \delta \ell}
\end{aligned}
$$

where $\eta$ is defined by root-mean-squared phonon fluctuations

$$
\begin{aligned}
\eta & \equiv \frac{G^{2}}{\delta \ell}\left\langle\left(u_{0}^{>}(z)\right)^{2}\right\rangle_{0} \\
& =\frac{G^{2}}{2 \pi \delta \ell} \frac{k_{B} T}{\sqrt{K B}} \int_{G_{z} e^{-\delta \ell}}^{G_{z}} \frac{d q_{z}}{\left|q_{z}\right|}
\end{aligned}
$$




$$
\begin{aligned}
& =\frac{G^{2}}{2 \pi} \frac{k_{B} T}{\sqrt{K B}} \\
& =\frac{k_{B} T}{\pi \epsilon_{0}},
\end{aligned}
$$

Because of the aforementioned relation of our model to a variety of other problems in condensed matter physics, this RG analysis is in fact quite familiar from those other contexts $^{38,44,46,47}$. As advertised it clearly predicts a phase transition at $\eta=2,{ }^{35}$ corresponding to

$$
\begin{aligned}
k_{B} T_{p} & =4 \pi G^{-2} \sqrt{K B}, \\
& =2 \pi \epsilon_{0},
\end{aligned}
$$

between the pinned phase for $T<T_{p}$ and the depinned phase for $T>T_{p}$. The two phases are distinguished by the relevance and irrelevance of the pinning potential $v$, respectively. More physically, for $T>T_{p}$ thermal fluctuations of the vortex lattice at long scales effectively average away the effects of the pinning potential (but see below), reducing it relative to the elastic energy.

In contrast for low temperatures $T<T_{p}$, as indicated by the RG flow, Eq.4.5, the strength of the pinning potential, no matter how weak at the lattice scale $a_{z}$ grows relative to and becomes stronger than the typical elastic energy. Quite clearly, since the computation of the RG flow in Eq.4.5 is done perturbatively in $v$ the flow terminates on scale $\xi(T) \equiv a_{z} e^{\ell_{*}}$ when elastic and pinning energies become comparable. On scales longer than $\xi(T)$, the displacement field is well-localized at a minimum of the periodic pinning potential, which therefore can be safely Taylor-expanded to quadratic order and treated as a "mass" for $u_{0}(z)$. Hence $\xi$ is determined by the balance of the effective pinning and elastic energies on scale $\xi$

$$
\frac{2 \sqrt{B K}}{\xi}\left(\frac{a}{2 \pi}\right)^{2}=v\left(\frac{\xi}{a_{z}}\right)^{-\eta / 2}
$$

with the effective pinning strength $v_{\text {eff }}=v\left(\xi / a_{z}\right)^{-\eta / 2} \leq$ $v$ reduced by thermal fluctuations of modes in the range of scales $a_{z}<z<\xi$. Solving Eq.4.9 for $\xi$ we find

$$
\xi(T) \approx \begin{cases}\xi_{0}, & \xi_{0} \lesssim a \sqrt{\frac{K}{B}} \\ \xi_{0}\left(\frac{\xi_{0}}{a} \sqrt{\frac{B}{K}}\right)^{\frac{\eta}{2-\eta}}, & \xi_{0}>a \sqrt{\frac{K}{B}}\end{cases}
$$

that, for weak short-scale pinning is, as expected, exponentially lengthened by thermal fluctuations

$$
\xi(T) \approx \xi_{0} e^{\sigma T /\left(T_{p}-T\right)}, \text { for } \xi_{0}>a \sqrt{\frac{K}{B}},
$$

with, $\sigma=\ln \left(\xi_{0} \sqrt{B / K} / a\right)>0$.

We next turn to the computation of correlation functions in each of these phases.

\section{B. Depinned phase, $T>T_{p}, h=0$}

Irrelevance of the pinning potential for $T>T_{p}$ allows us to compute correlation functions in the depinned phase perturbatively in $v$. To zeroth order, it is computed via a Gaussian integral with Hamiltonian $\mathcal{H}_{e l}$, Eq.2.1, at long scales giving

$$
\begin{aligned}
C_{T>T_{p}}(x, z) & =\left\langle(u(x, z)-u(0,0))^{2}\right\rangle, \\
& \approx 2 k_{B} T \int \frac{d q_{x} d q_{z}}{(2 \pi)^{2}} \frac{1-e^{i \mathbf{q} \cdot \mathbf{r}}}{B q_{x}^{2}+K q_{z}^{2}}, \\
& \approx \frac{k_{B} T}{\pi \sqrt{K B}} \ln \left[a^{-1} \sqrt{x^{2}+\frac{B}{K} z^{2}}\right],
\end{aligned}
$$

where we used vortex lattice spacing $a$ as a natural shortscale cutoff on $x$. At the location of the defect at $x=$ $0, C_{T>T_{p}}(0, z)$ can be equivalently computed using the effective 1D Hamiltonian $\mathcal{H}_{0}$, Eq.2.11, to zeroth order $(v=0)$ giving

$$
\begin{aligned}
C_{T>T_{p}}(0, z) & =\left\langle\left(u_{0}(z)-u_{0}(0)\right)^{2}\right\rangle \\
& \approx \frac{k_{B} T}{\pi \sqrt{K B}} \int_{0}^{G_{z}} \frac{d q_{z}}{q_{z}}\left(1-\cos q_{z} z\right), \\
& \approx \frac{k_{B} T}{\pi \sqrt{K B}} \ln \left(\frac{|z|}{a} \sqrt{\frac{B}{K}}\right)
\end{aligned}
$$

Vortex density correlations can be similarly computed using Eq.2.3 and phonon correlations above. To zeroth order in $v$, the average density is given

$$
\begin{aligned}
\langle n(x, z)\rangle_{0} & =n_{0}-n_{0}\left\langle\partial_{x} u\right\rangle_{0}+2 n_{G} \operatorname{Re}\left\langle e^{i 2 \pi n_{0}(x+u(x, z))}\right\rangle_{0}, \\
& \approx n_{0}+\left(\frac{a}{L} \sqrt{\frac{K}{B}}\right)^{\eta / 2} 2 n_{G} \cos \left(2 \pi n_{0} x\right), \\
& \approx n_{0},
\end{aligned}
$$

with fluctuations vanishing in the thermodynamic $(L \rightarrow$ $\infty)$ limit. Physically we do not expect this to be the case, as the pinning potential breaks translational invariance in $x$, even when it is irrelevant. This reveals that for $T>T_{p}$ the pinning potential is in fact dangerously irrelevant, as dropping it completely (as in above calculation) restores translational invariance and gives a result that is not even qualitatively correct for $\langle n(x, z)\rangle$. Indeed to correctly capture the behavior of $\langle n(x, z)\rangle$, we need to compute it to at least 1st order in $v$. The irrelevance of $v$ for $T>T_{p}$ guarantees the convergence of such perturbation theory. We find ${ }^{35}$

$$
\begin{aligned}
\langle n(x, z)\rangle-n_{0} & \approx 2 \frac{n_{G} v}{k_{B} T} \operatorname{Re} \int d z^{\prime}\left\langle e^{i G(x+u(x, z))} \cos \left[G u\left(0, z^{\prime}\right)\right]\right\rangle_{0} \\
& \approx \frac{n_{G} v}{k_{B} T} \cos G x \int d z^{\prime} e^{-\frac{G^{2}}{2}\left\langle\left[u(x, z)-u\left(0, z^{\prime}\right)\right]^{2}\right\rangle_{0}}, \\
& \approx c_{\eta} \frac{n_{G} v a}{k_{B} T} \sqrt{\frac{K}{B}}\left(\frac{a}{|x|}\right)^{\eta-1} \cos \left(2 \pi n_{0} x\right),
\end{aligned}
$$


$\left(c_{\eta}=O(1)\right.$ dimensionless constant $)$ showing that even in the phase where it is irrelevant, the pinning potential leads to Friedel oscillations in the density, a result missed by simply setting $v=0$. This illustrates often under-appreciated distinction between irrelevance in the
RG sense and unimportance of an operator in the physical sense.

Density two-point correlations can also be straightforwardly computed. Using above phonon correlations and Eq.2.3, we find ${ }^{35}$

$$
\left\langle n(x, z) n\left(x^{\prime}, 0\right)\right\rangle \approx\langle n(x, z)\rangle_{0}\left\langle n\left(x^{\prime}, 0\right)\right\rangle_{0}+\frac{n_{0}^{2} k_{B} T}{2 \pi} \sqrt{\frac{K}{B}} \frac{B z^{2}-K\left(x-x^{\prime}\right)^{2}}{\left[K\left(x-x^{\prime}\right)^{2}+B z^{2}\right]^{2}}+2 n_{G}^{2}\left(\frac{a^{2}}{\left(x-x^{\prime}\right)^{2}+\frac{B}{K} z^{2}}\right)^{\eta / 2} \cos \left(2 \pi n_{0} x\right)
$$

with the first term given by Eq.4.17, and with the last term approximated by its lowest order (in $v$ ) translationally invariant expression.

\section{Pinned phase, $T<T_{p}, h=0$}

In the low temperature pinned phase, $v$ is relevant, growing with increasing length scale relative to the elastic energy. On scales longer than $\xi$, we can therefore approximate its strong pinning effects by replacing it by a Dirichlet boundary condition on $u(x, z)$ at the location of the pin away from its ends (for a more systematic justification of this, see Appendix B), namely taking

$$
u(0, z)=u_{0}(z)=0 .
$$

Bulk phonon and density correlations with this boundary condition can still be computed exactly. As derived for a general case in Appendix B, a pinned phonon correlation function can be expressed in terms of a pin-free correlation function, i.e., from a purely harmonic elastic theory with $v=0$ :

$$
\begin{aligned}
G_{T<T_{p}}\left(x, x^{\prime} ; z\right) & =\left\langle u(x, z) u\left(x^{\prime}, 0\right)\right\rangle, \\
& =G_{0}\left(\left|x-x^{\prime}\right|, z\right)-G_{0}\left(|x|+\left|x^{\prime}\right|, z\right) .
\end{aligned}
$$

where $G_{0}(x, z)$ is $v=0$ two-point phonon correlation function. As expected, because of the pin at $x=0$, $G_{T<T_{p}}\left(x, x^{\prime} ; z\right)$ is clearly not translationally invariant in $x$, depending on both $x$ and $x^{\prime}$. It is easy to see that it vanishes identically for $x$ and $x^{\prime}$ on opposite sides of the pin, e.g., $x>0$ and $x^{\prime}<0$, showing that because of the pin such phonon fluctuations are completely uncorrelated on length scales longer than $\xi$. On the other hand, for $x$ and $x^{\prime}$ on the same side of the pin (e.g., $x>0$ and $\left.x^{\prime}>0\right)$, above expression is given by

$$
\begin{aligned}
G_{T<T_{p}}\left(x, x^{\prime} ; z\right) & =G_{0}\left(\left|x-x^{\prime}\right|, z\right)-G_{0}\left(x+x^{\prime}, z\right), \text { for } x>0, x^{\prime}>0, \\
& =\frac{1}{2}\left\langle\left[u(x, z)-u\left(-x^{\prime}, 0\right)\right]^{2}\right\rangle_{0}-\frac{1}{2}\left\langle\left[u(x, z)-u\left(x^{\prime}, 0\right)\right]^{2}\right\rangle_{0}, \\
& =\frac{k_{B} T}{4 \pi \sqrt{K B}} \ln \left[\frac{K\left(x+x^{\prime}\right)^{2}+B z^{2}}{K\left(x-x^{\prime}\right)^{2}+B z^{2}}\right],
\end{aligned}
$$

and at the same point $\left(x=x^{\prime}, z=z^{\prime}\right)$, reduces to

$$
\begin{aligned}
G_{T<T_{p}}(x, x ; 0) & =G_{0}(0,0)-G_{0}(2 x, 0), \\
& =\frac{1}{2}\left\langle[u(x, 0)-u(-x, 0)]^{2}\right\rangle_{0}, \\
& \approx \frac{k_{B} T}{2 \pi \sqrt{K B}} \ln \left(\frac{2|x|}{\bar{a}}\right) .
\end{aligned}
$$

In above, we naturally cut off separation at short scales by $\bar{a} \equiv \operatorname{Max}[a, \xi \sqrt{B / K}]$, since the Dirichlet boundary condition, Eq.4.19 is only valid on scales longer than $\bar{a}$. Utilizing this result, we can easily compute the average vortex density at point $x, z$

$$
\langle n(x, z)\rangle \approx n_{0}+2 n_{G}\left(\frac{\bar{a}}{2|x|}\right)^{\eta / 2} \cos \left(2 \pi n_{0} x\right) .
$$

$\langle n(x, z)\rangle$ in the pinned phase also displays Friedel oscillations similar to that of the depinned phase, Eq.4.16,4.17, but with an amplitude that is nonperturbative in $v$ and power-law fall-off with exponent $\eta / 2$, rather than $\eta-1$. 
The latter is continuous at the roughening transition, where $\eta\left(T_{p}\right) / 2=\eta\left(T_{p}\right)-1=1$. This is in agreement with the result first found by Affleck, et al. by utilizing an equivalent Luttinger liquid phenomenology ${ }^{35}$.

\section{Finite $T$ and finite tilt-field $h$}

The calculations of previous sections have focused on either thermal properties at a vanishing transverse field, or on finite transverse field response at a vanishing temperature. As we show below, these can be extended to a general point $T>0, h>0$ on the phase diagram, Fig.3.

\section{Finite $T$ tilting transition}

The zero-temperature results for the tilting transition of Sec.III can be extended to a finite temperature by utilizing RG analysis and matching from Sec.IV A. As in a related sine-Gordon commensurate-incommensurate transitions ${ }^{42,43}$ the basic nature of the transition remains the same but with thermally renormalized effective parameters. The extension of the zero-temperature transverse field boundary, Eq.3.22 to a finite-temperature phase boundary $h_{c 1}(T)$ is determined by the difference in free-energies of the commensurate and a single-soliton states, computed for $T<T_{p}$. The former is simply given by the sum of the energy of the commensurate state $E_{C}$ and the entropic free-energy contribution $F_{u}=-k_{B} T \ln \Omega_{u}$ of the phonon modes.

The computation of a single-soliton free energy is more complicated, since at a low temperature the pinning potential is relevant and therefore requires a strongcoupling analysis. This can be done by matching freeenergy calculations on scales shorter and longer than the strong coupling correlation length $\xi(T)$. As is clear from the analysis leading to $\xi(T)$, Eq.4.10, for $\xi_{0} \gg a_{z}$ (corresponding to weak pinning $v$ ) the pinning potential is subdominant on scales $a_{z}<z<\xi(T)$ and the free-energy is dominated by $F_{u}^{>}$due to Gaussian phonon fluctuations. On scales longer than $\xi(T)$, the pinning potential dominates and free-energy is determined by the sum of three contributions: (i) a single soliton energy $E_{1}(T)$, with short-scale cutoff $\xi(T)$ replacing $\xi_{0}$ in Eq.3.19, (ii) soliton's positional entropic contribution $-k_{B} T \ln (L / \xi(T))$, and (iii) free-energy contribution $F_{u}^{<}$due to phonon fluctuations about a single soliton configuration. Noting that phonon fluctuations about the commensurate and single soliton states are approximately the same, i.e., $F_{u} \approx F_{u}^{>}+F_{u}^{<}$, we find that the free energy difference between a single soliton and the commensurate state is given by:

$F_{s 1}(T) \approx E_{s 1}(T)-k_{B} T \ln \frac{L}{\xi(T)}$,

$$
\begin{aligned}
& \approx \epsilon_{0}\left[2 \pi \ln \frac{L}{\xi(T)}-4 \pi \frac{L}{a} h\right]-k_{B} T \ln \frac{L}{\xi(T)} \\
& \approx T_{p} \frac{2 L}{a}\left[\frac{a}{2 L}\left(1-\frac{T}{T_{p}}\right) \ln \frac{L}{\xi(T)}-h\right]
\end{aligned}
$$

This leads to the prediction for $h_{c 1}(T)$ quoted in the Introduction:

$$
\begin{aligned}
h_{c 1}(T) & \approx \frac{a}{2 L}\left(1-\frac{T}{T_{p}}\right) \ln \frac{L}{\xi(T)}, \\
& \approx h_{c 1}(0)\left(1-\frac{T}{T_{p}}\right) .
\end{aligned}
$$

In going from the first to second line we used a temperature-independent approximation $\xi_{0}$ for $\xi(T)$, Eq.4.10, valid for large $L$.

\section{Correlations at $T>0, h>0$}

Correlation functions at a finite $h$ and $T$ can also be computed. The nature of valid approximation and their resulting form strongly depends on three possible regimes on the phase diagram, Fig.3.

(a) Incommensurate (fully-tilted) state, $h>h_{c 2}(T)$ :

The strongly incommensurate, high tilt and temperature regime $h>h_{c 2}(T)$, where the effects of the pin can be treated perturbatively is simplest to analyze, as was first done in Ref. 35. This can be done in close analogy to Sec.IV B, extending it to a finite $h$. The governing Hamiltonian is given by

$$
\begin{aligned}
\mathcal{H}= & \frac{1}{2} \int d x d z\left[K\left(\partial_{z} \tilde{u}\right)^{2}+B\left(\partial_{x} \tilde{u}\right)^{2}\right] \\
& -v \int d z \cos [G(\tilde{u}(0, z)+h z)]
\end{aligned}
$$

with $\tilde{u}(x, z)$ fluctuating around 0 and to zeroth order in the pin strength $v$ exhibiting translationally invariant correlations:

$$
\left\langle(\tilde{u}(x, z)-\tilde{u}(0,0))^{2}\right\rangle_{0} \approx \frac{k_{B} T}{\pi \sqrt{K B}} \ln \left[a^{-1} \sqrt{x^{2}+\frac{B}{K} z^{2}}\right] .
$$

To the same zeroth order the average vortex density is uniform, $n_{0}$. To compute it to the lowest nontrivial order in $v$, we expand the Boltzmann weight to first order in the pinning potential, $v$ and utilize the phonon correlations of $\tilde{u}(x, z)$, Eq.4.32 about the tilted state. This leads to: 


$$
\begin{aligned}
\langle n(x, z)\rangle-n_{0} & \approx \frac{n_{G} v}{k_{B} T} \operatorname{Re}\left[e^{i G x} \int d z^{\prime} e^{-\frac{G^{2}}{2}\left\langle\left[\tilde{u}(x, z)-\tilde{u}\left(0, z^{\prime}\right)\right]^{2}\right\rangle_{0}} e^{i G h\left(z-z^{\prime}\right)}\right] \\
& \approx \frac{n_{G} v}{k_{B} T} \operatorname{Re}\left[e^{i G x} \int d z^{\prime}\left(\frac{a}{\sqrt{x^{2}+\frac{B}{K}\left(z-z^{\prime}\right)^{2}}}\right)^{\eta} e^{i G h\left(z-z^{\prime}\right)}\right], \\
& \approx \frac{n_{G} v a}{k_{B} T} \sqrt{\frac{K}{B}} g_{\eta}(|x| / a) e^{-|x| / \lambda_{h}^{\infty}} \cos \left(2 \pi n_{0} x\right),
\end{aligned}
$$

with the transverse field $h$ giving an exponential fall off of Friedel oscillations on scale $\lambda_{h}^{\infty}$, Eq.3.51, ${ }^{35}$ and preexponential function $g_{\eta}(|x| / a)$ approximated by a power-law

$g_{\eta}(|x| / a) \approx \begin{cases}c_{\eta}\left(\frac{a}{|x|}\right)^{\eta-1}, & a \ll|x| \ll \lambda_{h}^{\infty}, \\ d_{\eta}\left(\frac{a}{\lambda_{h}^{\infty}}\right)^{\eta / 2-1}\left(\frac{a}{2|x|}\right)^{\eta / 2}, & \lambda_{h}^{\infty} \ll|x|,\end{cases}$

$c_{\eta}, d_{\eta} \mathrm{O}(1)$ dimensionless constants.

The exponential decay of density correlations, Eq.4.33 vindicates a perturbative treatment (in $v$ ) at large transverse field and temperature, where the decay length $\lambda_{h}^{\infty}$ is the shortest scale in the problem.

(b) Commensurate (pinned) transverse Meissner state, $h<h_{c 1}(T)$ :

Vortex correlations are also simple to analyze in the low tilt-field, low-temperature commensurate $h<$ $h_{c 1}$ state, where, because of the commensurateincommensurate (tilting/roughening) transition we expect a behavior that is qualitatively different from that of the incommensurate state. We expand the Hamiltonian, Eq.4.31 in vortex lattice displacements $w(x, z)$ about the nontrivial zero-temperature distortion $u_{0}(x, z)$, Eq.3.48 (with $n_{s}=0$ ) characterizing this state:

$$
\tilde{u}(x, z)=\tilde{u}_{0}(x, z)+w(x, z) .
$$

To quadratic order in $w$ we find

$$
\begin{aligned}
\delta \mathcal{H} & =\int d x d z\left[\frac{K}{2}\left(\partial_{z} w\right)^{2}+\frac{B}{2}\left(\partial_{x} w\right)^{2}\right] \\
& +\frac{1}{2} v\left(2 \pi n_{0}\right)^{2} \int d z w(0, z)^{2},
\end{aligned}
$$

where we used the fact that in the commensurate state $u_{0}(0, z)=0$. Clearly then, because the pinning nonlinearity is localized at $x=0$, vortex correlations of $w(x, z)$ on top of the ground state background $u_{0}(x, z)$ are identical to those of the $h=0$ state. Namely, on scales longer than the pinning length $\xi, w(x, z)$ fluctuations are that of a field that is harmonic in the bulk, but pinned at the $x=0$ boundary. This identification allows us to take over results from Sec.IV C. Using the simplified version for $u_{0}(x, z)$, Eq.3.49, we therefore find the average vortex density

$$
\begin{aligned}
\langle n(x, z)\rangle & =n_{0}-n_{0} \partial_{x} u_{0}(x, z)+2 n_{G} \operatorname{Re}\left[e^{i G\left(x+u_{0}(x, z)\right)}\left\langle e^{i G w(x, z)}\right\rangle\right] \\
& \approx n_{0}-n_{0} \sqrt{\frac{K}{B}} \frac{\pi}{L} h z \operatorname{sgn}(x) e^{-\sqrt{\frac{K}{B}} \frac{\pi}{L}|x|}+2 n_{G}\left(\frac{\bar{a}}{2|x|}\right)^{\eta / 2} \cos \left[2 \pi n_{0}\left(x+u_{0}(x, z)\right)\right],
\end{aligned}
$$

that exhibits power-law decaying Friedel oscillations (non-perturbative in $v$ ) as well as a smooth compression and dilation of the vortex lattice around the pin for $z \neq 0$. Both features can be clearly seen in the vortex configuration displayed in Fig.13.

(c) Incommensurate (soliton-tilted) state, $h_{c 1}(T)<$ $h \ll h_{c 2}(T)$ :

We now turn to the intermediate incommensurate regime of $h$ and $T$, with $h>h_{c 1}(T)$. Because there is only a single CI transition (that across $h_{c 1}$, with $h_{c 2}$ simply a crossover), we do not expect any qualitative change in correlations from those found above for large $h$ and $T$. However, examining the expression for $\langle n(x, z)\rangle$, Eqs.4.33, 4.34, the prefactor $\left(a / \lambda_{h}^{\infty}\right)^{\eta / 2-1}$, that is large for $\eta<2$ and $\lambda_{h}^{\infty} \gg a$, suggests a failure of the perturbative treatment for $T<T_{p}$ and for a sufficiently low $h$. Indeed, as illustrated in Fig.17, in such a regime $\lambda_{h}^{\infty}$ will exceed the pinning length $\xi \sqrt{B / K}$. Since for 
$T<T_{p}$, on scales longer than $\xi \sqrt{B / K}$, even a (bare) weak pinning potential becomes comparable to the elastic energy and therefore cannot be treated perturbatively. Consequently, for $\xi \sqrt{B / K}<\lambda_{h}^{\infty}$ there opens up an intermediate regime, $\xi \sqrt{B / K}<x<\lambda_{h}^{\infty}$, where above perturbative (in $v$ ) analysis leading to exponential decay of Friedel oscillations, Eqs.4.33, 4.34 fails. The crossover boundary $h_{*}(T)$ separating the perturbative and nonperturbative regimes is clearly given by $\xi \sqrt{B / K}=\lambda_{h}^{\infty}$, which (not surprisingly, but reassuringly) is equivalent to $h_{*}(T) \approx h_{c 2}(T) \approx a / 2 \pi \xi(T)$ found in Eq.3.36.

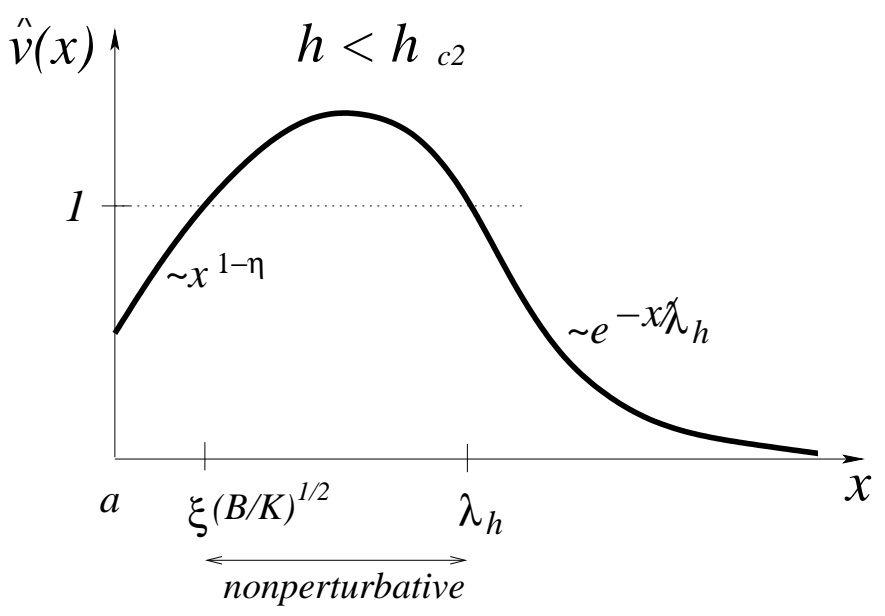

FIG. 17: A schematic of the length-scale dependent pinning coupling $\hat{v}(x)$, indicating its intermediate nonperturbative regime for $h<h_{c 2}(T)$.

As schematically illustrated in Fig.17, in the parlance of RG, for $T<T_{p}$ the dimensionless pinning coupling $\hat{v}(x)$ at short scale $x$ grows as $x^{1-\eta / 2}$. For $h>h_{c 2}(T)$, before $\hat{v}(x)$ reaches a nonperturbative regime (i.e., reaches $O(1)$ ), this growth is "quenched" by the tilt field $h$ on scale longer than $\lambda_{h}^{\infty}$, beyond which $\hat{v}(x) \sim e^{-x / \lambda_{h}^{\infty}}$ decreases exponentially, validating the fully perturbative treatment of case (a) above. In contrast, for $h<h_{c 2}(T)$, $\hat{v}(x)$ becomes large on scale $\xi \sqrt{B / K}<\lambda_{h}^{\infty}$, requiring a nonperturbative analysis on longer scales.

Above discussion clearly indicates a need for separate treatments of three distinct regimes of length scales (i) $a<x<\xi \sqrt{B / K}$, (ii) $\xi \sqrt{B / K}<x<\lambda_{h}$, (iii) $\lambda_{h}<x$.
In the regime (i) of shortest lengths scales, the pinning potential in $\mathcal{H}$, Eq.4.31 can be treated perturbatively, with average density given by the $x \ll \lambda_{h}^{\infty}$ limit of the result in Eq.4.33, with exponential factor approximately 1. On longer scales, in regime (ii) the pinning potential is comparable to the elastic energy and vortex configurations displays a soliton array of density $n_{s}(h)$, illustrated in Fig.15. In this regime $\xi \sqrt{B / K}<x<\lambda_{h}^{\infty}<\lambda_{h}$ segments of vortex lines on the corresponding scales shorter than $n_{s}^{-1}$ appear to be strongly pinned in the vicinity of the columnar defect. Hence on these scales we expect vortex correlations of the transverse Meissner state computed in Eq.4.38, with only small corrections from soliton array fluctuations. Finally, on scales $z>n_{s}^{-1}$ (corresponding to $x>\lambda_{h}$ of regime (iii)), it is clear from Fig.15, that vortex lattice displays an average tilt $a n_{s}(h)$,

$$
u(x, z)=a n_{s} z+\tilde{w}(x, z),
$$

with small fluctuations $\tilde{w}(x, z)$ about the soliton state arising from vibration of the soliton array. We expect the corresponding effective Hamiltonian for $\tilde{w}(x, z)$ to be given by

$$
\begin{aligned}
\delta \overline{\mathcal{H}}= & \frac{1}{2} \int d x d z\left[\bar{K}\left(\partial_{z} \tilde{w}\right)^{2}+\bar{B}\left(\partial_{x} \tilde{w}\right)^{2}\right] \\
& -\bar{v} \int d z \cos \left(G\left(\tilde{w}(0, z)+a n_{s} z\right)\right)
\end{aligned}
$$

with $\bar{K} \approx K, \bar{B} \approx B$ the effective moduli of the solitontilted vortex lattice and $\bar{v} \approx v\left(\xi / a_{z}\right)^{-\eta / 2}$ a weak pinning potential, reduced by thermal fluctuations from scales $a_{z}<z<\xi$ of regime (ii). On longer scales $z>n_{s}^{-1}$ (regime (iii)), the soliton spacing $n_{s}^{-1}$ is the shortest scale in the problem (beyond the lattice spacing), and analysis of $w$ correlations using $\delta \overline{\mathcal{H}}$ can be done perturbatively in $\bar{v}$ similar to that of regime (a), that led to Eq.4.33. As can be seen from comparing Hamiltonians in Eq.4.31 and Eq.4.40, the main qualitative difference is the replacement of $h$ by $a n_{s}(h)$ inside the pinning potential, that leads to the corresponding replacement of $\lambda_{h}^{\infty}(h)$ by the longer length $\lambda_{h}(h)$ in the exponential decay of Friedel oscillations of the average vortex density. Putting these results together for $h_{c 1}<h<h_{c 2}$ we predict

$$
\langle n(x, z)\rangle-n_{0} \sim \cos \left(2 \pi n_{0} x\right) \begin{cases}\frac{n_{G} v a}{k_{B} T} \sqrt{\frac{K}{B}}\left(\frac{a}{|x|}\right)^{\eta-1}, & a<|x|<\xi \sqrt{B / K}, \\ 2 n_{G}\left(\frac{a}{2|x|}\right)^{\eta / 2}, & \xi \sqrt{B / K}<|x|<\lambda_{h}, \\ \frac{n_{G} \bar{v} \lambda_{h}}{k_{B} T} \sqrt{\frac{K}{B}}\left(\frac{\lambda_{h}}{|x|}\right)^{\eta-1} e^{-|x| / \lambda_{h}}, & \lambda_{h}<|x| .\end{cases}
$$

\section{FINITE DENSITY OF INDEPENDENT COLUMNAR DEFECTS}

So far we have focused on an idealized problem of a single columnar defect. As discussed in the Introduc- tion, no genuine tilting CI phase transition is possible 
in this case, since pinning energy density and the associated lower critical field $h_{c 1}$, Eq.3.22 vanish in the 2D bulk thermodynamic limit. In this section we extend our results to a physically more interesting case of a finite dilute concentration $1 / d$ of columnar defects.

A full treatment of such a highly nontrivial problem is beyond the scope of the present paper and has been a subject of numerous studies. ${ }^{17,18,21-24}$ Here we will be content with an approximate analysis of a dilute concentration of columnar defects on intermediate length scales, where vortex lattice response around each pin can be treated independently. However, we expect that for a fixed defect concentration (even if dilute), the system will crossover to the anisotropic (Bose) vortex glass collectivepinning phenomenology and our results will break down on sufficiently long scales. This is in the spirit of other problems in physics, most notably the Kondo effect ${ }^{47}$, where for a sufficiently dilute concentration of impurities, on intermediate scales local moments can be treated independently, but may order magnetically at sufficiently long scales and low temperatures.

The main qualitative effect of a finite pin spacing $d$ should be clear from the analysis of Sec.III D, in particular from Eqs.2.10, 3.48 and vortex configuration illustrated in Fig.15. There we have shown that a vortex lattice distortion of wavelength $\lambda_{z}$ along the defect penetrates to length $\lambda_{\perp}=\lambda_{z} \sqrt{B / K} / 2 \pi$ along $x$ into the bulk, away from the defect. For a finite concentration of columnar defects, we therefore expect long wavelength elastic distortions with $\lambda_{z}>2 \pi d \sqrt{K / B}$, corresponding to overlapping distortion clouds of neighboring pins to be cut off at scale $d$ along $x$.

To demonstrate this in detail, we need to generalize our results in Eqs.2.10, 3.48 to a nontrivial boundary condition on the elastic distortion $u(x, z)$ at $x=d$, and use it to recalculate predictions of previous sections, most importantly the energies of the commensurate (aligned) and incommensurate (soliton) states. The correct qualitative physics that we are after here can be obtained by the use of periodic boundary condition on $u(x, z)$ with period $d$, or even more simply (and a bit cruder) a boundary condition of simply cutting off $x$ integrals beyond length $d / 2$ around each pin, corresponding to the Dirichlet boundary condition on $u(x, z)$.

To see this in more detail, we recalculate the energy per columnar defect $E=\mathcal{H}\left[u_{0}(x, z)\right]$, Eq.2.5,

$$
\begin{aligned}
E= & \frac{1}{2} \int_{0}^{L} d z \int_{-d / 2}^{d / 2} d x\left[K\left(\partial_{z} u_{0}-h\right)^{2}+B\left(\partial_{x} u_{0}\right)^{2}\right] \\
& -v \int_{0}^{L} d z \cos \left(G u_{0}(0, z)\right)
\end{aligned}
$$

with the above boundary condition at $|x|=d / 2$ and $u_{0}(x, z)$ given by Eq.3.48. Since Euler-Lagrange equation ensures that contributions from each of the three terms balance each other, $E$ approximately reduces to

$$
E \approx \int_{0}^{L} d z \int_{0}^{d / 2} d x K\left[\partial_{z} \bar{u}_{0}(x, z)-h_{\mathrm{eff}} e^{-\sqrt{\frac{K}{B}} \frac{\pi}{L}|x|}\right]^{2}-v L
$$

Calculating above expression we find, that, for $L<$ $2 \pi d \sqrt{K / B}$ it reduces to the previously calculated singlepin result given in Eq.3.32. In the opposite regime, $L>2 \pi d \sqrt{K / B}$ of interest to us, $E$ crosses over to

$$
E(d) \approx E_{C}(d)+K a d\left(h_{c 1}-h\right) N_{s}+\frac{1}{2} K a^{2} \frac{d}{L} N_{s}^{2},
$$

with bulk energy per pin

$$
E_{C}(d)=K h^{2} L d-v L,
$$

leading to

$$
h_{c}(d)=\sqrt{\frac{v}{K d}}
$$

and

$$
h_{c 1}(d) \approx \sqrt{\frac{B}{K}} \frac{a}{4 \pi d} \ln \left[\sqrt{\frac{K}{B}} \frac{2 \pi d}{\xi}\right],
$$

as quoted in the Introduction.

\section{CONCLUSIONS}

In this paper we studied a finite-temperature response of a planar vortex array to an in-plane tilting of an external magnetic field away from a dilute concentration of pinning columnar defects. We found that the vortex lattice tilting proceeds via an interesting finite temperature, finite transverse field commensurateincommensurate transition at $h_{c 1}(T)$, driven by a proliferation of solitons, as illustrated in the phase diagram, Fig.3. A sensitive dependence of this lower-critical field (that vanishes for a single pin) on the columnar defect spacing should be experimentally testable by varying the heavy-ion irradiation flux used to create pinning tracks. We show that at low-temperatures, for $h>h_{c 1}(T)$ the vortex array exhibits a highly nontrivial soliton-like distortion as a compromise between inter-vortex interaction, and the pinning and diamagnetic energies. We show that this nonlinear response persists up to a large uppercritical tilting angle, $\tan \theta_{c 2} \sim a / \xi(T)$, beyond which system recovers a full linear transverse susceptibility with $B_{\perp} \approx H_{\perp}$. We expect that these and many other detailed predictions should be directly testable in computer simulations. Although much more difficult, it is our hope that the theory presented here can be furthermore tested in mesoscopic samples of artificially layered superconductors.

More importantly, we expect that a number of these features will carry over to a transverse field response in bulk superconductors. Extending our two-dimensional planar results and exploring their impact on phenomenology of bulk samples remains an important and challenging problem. 


\section{Acknowledgments}

It is a pleasure to acknowledge illuminating discussions with M. Ablowitz, I. Affleck, V. Gurarie, D. R. Nelson, A. Polkovnikov, and J. Toner. I thank an anonymous referee for a careful reading of the manuscript, making valuable suggestions, and catching a number of typographical errors. This research was support by the National Science Foundation through MRSEC DMR-0213918, DMR0321848, and by the David and Lucile Packard Foundation.

\section{APPENDIX A: BOUNDARY HAMILTONIAN VIA A FUNCTIONAL INTEGRAL}

Problems where it is possible and convenient to eliminate bulk degrees of freedom, thereby reducing the problem to a lower-dimensional one are quite common in physics. In this appendix, for completeness, we present a general functional-integrals approach to such problems, and apply it to the problem of a planar vortex lattice pinned by a single columnar defect treated my more pedestrian methods in the main text.

To this end, we consider a field $\phi(\mathbf{x}, \mathbf{z})$ defined on a coordinate space $\mathbf{r}=(\mathbf{x}, \mathbf{z})$ that we split into bulk $\mathbf{x}$ and boundary $\mathbf{z}$ subspaces. The energetics is governed by a Hamiltonian $\mathcal{H}[\phi]$ and the partition function is given by a standard functional integral over the field $\phi(\mathbf{r})\left(k_{B} T \equiv\right.$ $1)$ :

$$
Z=\int[d \phi(\mathbf{r})] e^{-\mathcal{H}[\phi]}
$$

$Z$ can be equivalently expressed as an integral over field $\phi(\mathbf{r})$ constrained on the boundary $\mathbf{x}=0$ to be $\phi(\mathbf{0}, \mathbf{z})=$ $\phi_{0}(\mathbf{z})$, followed by an integral over the boundary fields $\phi_{0}(\mathbf{z})$. Explicitly, the former is implemented by a functional $\delta$-function, $\delta[\phi(\mathbf{z})] \equiv \prod_{\mathbf{z}} \delta(\phi(\mathbf{z}))$ giving

$$
\begin{aligned}
Z & =\int\left[d \phi_{0}(\mathbf{z}) d \phi(\mathbf{x}, \mathbf{z})\right] \delta\left[\phi(\mathbf{0}, \mathbf{z})-\phi_{0}(\mathbf{z})\right] e^{-\mathcal{H}[\phi]}, \\
& \equiv \int\left[d \phi_{0}(\mathbf{z})\right] e^{-\mathcal{H}_{0}\left[\phi_{0}(\mathbf{z})\right]}
\end{aligned}
$$

The effective boundary Hamiltonian $\mathcal{H}_{0}\left[\phi_{0}(z)\right]$ defined above can be expressed using the Fourier representation of the functional $\delta$-function, leading to

$$
e^{-\mathcal{H}_{0}\left[\phi_{0}(\mathbf{z})\right]}=\int[d \phi d \lambda] e^{-\mathcal{H}[\phi]+i \int_{\mathbf{z}} \lambda(\mathbf{z})\left(\phi(\mathbf{0}, \mathbf{z})-\phi_{0}(\mathbf{z})\right)} .
$$

For a general Hamiltonian $\mathcal{H}[\phi]$, above computation can only be performed via a formal cumulant expansion. However, for a special case of a quadratic Hamiltonian

$$
\mathcal{H}[\phi]=\frac{1}{2} \int_{\mathbf{r}, \mathbf{r}^{\prime}} \phi(\mathbf{r}) G^{-1}\left(\mathbf{r}-\mathbf{r}^{\prime}\right) \phi\left(\mathbf{r}^{\prime}\right),
$$

defined by a correlation function $G(\mathbf{r})$ (with $G^{-1}(\mathbf{r})$ its inverse), all cumulants reduce to a power of $G(\mathbf{r})$, i.e., obey Wick's theorem, equivalent to the Gaussian integral identity

$$
\int_{-\infty}^{\infty} d \phi e^{-\frac{1}{2} a^{-1} \phi^{2}+\lambda \phi}=\left(\frac{2 \pi}{a}\right)^{1 / 2} e^{\frac{1}{2} a \lambda^{2}} .
$$

A Gaussian functional integrations over $\phi(\mathbf{r})$ and $\lambda(\mathbf{z})$ (dropping an inconsequential $\phi_{0}$-independent constant) then gives

$$
\begin{aligned}
e^{-\mathcal{H}_{0}\left[\phi_{0}(\mathbf{z})\right]} & =\int[d \lambda] e^{-\frac{1}{2} \int_{\mathbf{z}, \mathbf{z}^{\prime}} \lambda(\mathbf{z}) G\left(\mathbf{0}, \mathbf{z}-\mathbf{z}^{\prime}\right) \lambda\left(\mathbf{z}^{\prime}\right)-i \int_{\mathbf{z}} \lambda(\mathbf{z}) \phi_{0}(\mathbf{z})} . \\
& =e^{-\frac{1}{2} \int_{\mathbf{z}, \mathbf{z}^{\prime}} \phi_{0}(\mathbf{z}) G^{-1}\left(\mathbf{0}, \mathbf{z}-\mathbf{z}^{\prime}\right) \phi_{0}\left(\mathbf{z}^{\prime}\right)} .
\end{aligned}
$$

Applying this simple result to the $(1+1)$-dimensional (planar) vortex lattice pinned by a columnar defect at $x=0$ leads to

$$
\mathcal{H}_{0}\left[u_{0}(z)\right]=\frac{1}{2} \int_{z, z^{\prime}} u_{0}(z) G_{0}^{-1}\left(z-z^{\prime}\right) u_{0}\left(z^{\prime}\right),
$$

with

$$
G_{0}^{-1}(z) \equiv G^{-1}(0, z)
$$

an inverse of the bulk propagator $G(0, z)$ evaluated at $x=0$. In $q_{z}$-Fourier space the latter is easily evaluated

$$
\begin{aligned}
\tilde{G}\left(x=0, q_{z}\right) & =\int \frac{d q_{x}}{2 \pi} \frac{1}{K q_{z}^{2}+B q_{x}^{2}}, \\
& =\frac{1}{2 \sqrt{B K}} \frac{1}{\left|q_{z}\right|},
\end{aligned}
$$

and leads to

$$
\tilde{G}_{0}^{-1}\left(q_{z}\right)=2 \sqrt{B K}\left|q_{z}\right|,
$$

thereby confirming the resulting for $\mathcal{H}_{0}\left[u_{0}\left(q_{z}\right)\right]$, Eq.2.12 obtained in Sec.II B by a different method.

\section{APPENDIX B: BULK CORRELATION FUNCTIONS IN A "PINNED" STATE}

In this appendix, as a model of phonon correlations in the "pinned" vortex state, we study bulk correlation function of a Gaussian field constrained at $\mathbf{r}=(0, \mathbf{z})$ by a "massive" boundary Hamiltonian $\mathcal{H}_{p}[\phi(\mathbf{x}, 0)]$. As argued in the main text, on sufficiently long scales the boundary Hamiltonian can simply be implemented as a hard constraint on the field to vanish at $\mathbf{r}=(0, \mathbf{z})$. To this end we compute the asymptotic generating function $Z[j(\mathbf{r})]$

$Z[j(\mathbf{r})]=\int[d \phi(\mathbf{x}, \mathbf{z})] \delta[\phi(\mathbf{0}, \mathbf{z})] e^{-\mathcal{H}[\phi]+\int_{\mathbf{r}} j(\mathbf{r}) \phi(\mathbf{r})}$,

from which, by differentiation with respect to $j(\mathbf{r})$ all $n$-point correlation functions of $\phi(\mathbf{r})$ can be obtained. 
As in Appendix A, we have implemented the Dirichlet boundary condition on $\phi(\mathbf{r})$ via a functional $\delta$-function. Representing the latter in its (functional) Fourier form as in Eq.A4, using harmonic Hamiltonian, Eq.A5, and performing a Gaussian integral over $\phi(\mathbf{r})$, we find

$$
Z[j(\mathbf{r})]=e^{\frac{1}{2} \int_{\mathbf{q}, \mathbf{q}^{\prime}} j(\mathbf{q}) \Gamma\left[\mathbf{q}, \mathbf{q}^{\prime}\right] j\left(\mathbf{q}^{\prime}\right)}
$$

The kernel $\Gamma\left[\mathbf{q}, \mathbf{q}^{\prime}\right]$ is given by

$$
\Gamma\left[\mathbf{q}, \mathbf{q}^{\prime}\right]=(2 \pi)^{d_{z}} \delta^{d_{z}}\left(\mathbf{q}_{z}+\mathbf{q}_{z}^{\prime}\right) \tilde{G}\left(\mathbf{q}_{x}, \mathbf{q}_{z}\right)\left[(2 \pi)^{d_{x}} \delta^{d_{x}}\left(\mathbf{q}_{x}+\mathbf{q}_{x}^{\prime}\right)-\tilde{G}\left(\mathbf{q}_{x}^{\prime},-\mathbf{q}_{z}\right)\left(\int_{\mathbf{q}_{x}^{\prime \prime}} \tilde{G}\left(\mathbf{q}_{x}^{\prime \prime}, \mathbf{q}_{z}\right)\right)^{-1}\right]
$$

and leads to real-space $\phi(\mathbf{r}) 2$-point correlation function

$$
G_{\text {pinned }}\left[\mathbf{x}, \mathbf{x}^{\prime} ; \mathbf{z}\right]=\left\langle\phi(\mathbf{x}, \mathbf{z}) \phi\left(\mathbf{x}^{\prime}, 0\right)\right\rangle_{\text {pinned }},
$$

$$
=\frac{e^{-\left(\frac{K}{B}\right)^{\frac{1}{2}}\left|q_{z}\right||x|}}{2 \sqrt{B K}\left|q_{z}\right|}
$$

that in $\mathbf{q}_{z}$-Fourier space is given by

$$
\tilde{G}_{\text {pinned }}\left[\mathbf{x}, \mathbf{x}^{\prime} ; \mathbf{q}_{z}\right]=\tilde{G}\left(\mathbf{x}-\mathbf{x}^{\prime} ; \mathbf{q}_{z}\right)-\frac{\tilde{G}\left(\mathbf{x}, \mathbf{q}_{z}\right) \tilde{G}\left(\mathbf{x}^{\prime},-\mathbf{q}_{z}\right)}{\tilde{G}\left(0, \mathbf{q}_{z}\right)}
$$

In the special $(1+1)$-dimensional case of $\tilde{G}^{-1}\left(q_{x}, q_{z}\right)=$ $K q_{z}^{2}+B q_{x}^{2}$ of interest to us in the main text,

$$
\tilde{G}\left(x, q_{z}\right)=\int \frac{d q_{x}}{2 \pi} \frac{e^{i q_{x} x}}{K q_{z}^{2}+B q_{x}^{2}}, \quad \text { (B6) and leads to }
$$

$$
\begin{aligned}
G_{\text {pinned }}\left[x, x^{\prime} ; z\right] & =\frac{1}{2 \sqrt{B K}\left|q_{z}\right|}\left[e^{-\left(\frac{K}{B}\right)^{\frac{1}{2}}\left|q_{z}\right|\left|x-x^{\prime}\right|}-e^{\left.-\left(\frac{K}{B}\right)^{\frac{1}{2}}\left|q_{z}\right|\left(|x|+\left|x^{\prime}\right|\right)\right]}\right. \\
& =G\left(\left|x-x^{\prime}\right|, z\right)-G\left(|x|+\left|x^{\prime}\right|, z\right) .
\end{aligned}
$$

utilized in Eq.4.23 of the main text.

A simpler way to derive above "pinned" correlation function result is to note that the boundary condition $\phi(\mathbf{0}, \mathbf{z})=0$ is automatically explicitly satisfied by the odd part of $\phi(\mathbf{x}, \mathbf{z})$. It is also satisfied by a subset of (an independent) even part of $\phi(\mathbf{x}, \mathbf{z})$ that vanishes at $\mathbf{x}=\mathbf{0}$. Naively, such even/odd field decomposition does not represent a simplification since a constraint on the even part must still be enforced. However, it is clear that for correlations on the same side of the pin, a constrained even part of the field has identical correlations to that of the odd part of the field. Hence, $G_{\text {pinned }}\left[\mathbf{x}, \mathbf{x}^{\prime} ; \mathbf{z}\right]$ for $\mathbf{x} \mathbf{x}^{\prime}>0$ (i.e., on the same side of the pin) is simply given by twice the correlator of the unconstrained odd part, $\phi(\mathbf{x}, \mathbf{z}) \rightarrow \frac{1}{2}(\phi(\mathbf{x}, \mathbf{z})-\phi(-\mathbf{x}, \mathbf{z}))$

$$
\begin{aligned}
G_{\text {pinned }}\left[\mathbf{x}, \mathbf{x}^{\prime} ; \mathbf{z}\right] & =\frac{1}{2}\left\langle(\phi(\mathbf{x}, \mathbf{z})-\phi(-\mathbf{x}, \mathbf{z}))\left(\phi\left(\mathbf{x}^{\prime}, 0\right)-\phi\left(-\mathbf{x}^{\prime}, 0\right)\right)\right\rangle_{0}, \\
& =\left\langle\phi(\mathbf{x}, \mathbf{z}) \phi\left(\mathbf{x}^{\prime}, 0\right)\right\rangle_{0}-\langle\phi(\mathbf{x}, \mathbf{z}) \phi(-\mathbf{x}, 0)\rangle_{0},
\end{aligned}
$$

giving the result in Eq.B9.

We conclude this appendix with a computation of the generating function $Z[j(\mathbf{r})]$, that extends above analysis 
to scales shorter than the pinning length. To this end, we supplement the harmonic bulk Hamiltonian with a pinning one at the boundary, $\mathcal{H}_{p}[\phi(0, \mathbf{z})]$ with the full $\mathcal{H}$ given by

$\mathcal{H}[\phi]=\frac{1}{2} \int_{\mathbf{r}, \mathbf{r}^{\prime}} \phi(\mathbf{r}) G^{-1}\left(\mathbf{r}-\mathbf{r}^{\prime}\right) \phi\left(\mathbf{r}^{\prime}\right)+\int_{\mathbf{r}} \delta^{d_{x}}(\mathbf{x}) \mathcal{H}_{p}[\phi(0, \mathbf{z})]$,
The corresponding generating function is then given by

$$
Z[j(\mathbf{r})]=\int\left[d \phi_{0}(\mathbf{z})\right] e^{-\mathcal{H}_{p}\left[\phi_{0}(\mathbf{z})\right]} \int[d \phi(\mathbf{x}, \mathbf{z})] \delta\left[\phi(\mathbf{0}, \mathbf{z})-\phi_{0}(\mathbf{z})\right] e^{-\frac{1}{2} \int_{\mathbf{r}, \mathbf{r}^{\prime}} \phi(\mathbf{r}) G^{-1}\left(\mathbf{r}-\mathbf{r}^{\prime}\right) \phi\left(\mathbf{r}^{\prime}\right)+\int_{\mathbf{r}} j(\mathbf{r}) \phi(\mathbf{r})} .
$$

As in Appendix A, representing the functional $\delta$-function in its Fourier form, integrating over the harmonic bulk and boundary Fourier fields $\phi(\mathbf{r}), \lambda(\mathbf{z})$, we find

$$
Z[j(\mathbf{r})]=\int\left[d \phi_{0}(\mathbf{z})\right] e^{-\mathcal{W}\left[\phi_{0}(\mathbf{z}), j(\mathbf{r})\right]},
$$

with

$$
\begin{aligned}
\mathcal{W}\left[\phi_{0}, j\right] & =H_{p}\left[\phi_{0}(\mathbf{z})\right]-\frac{1}{2} \int_{\mathbf{r}, \mathbf{r}^{\prime}} j(\mathbf{r}) G\left(\mathbf{r}-\mathbf{r}^{\prime}\right) j\left(\mathbf{r}^{\prime}\right) \\
& +\frac{1}{2} \int_{\mathbf{z}, \mathbf{z}^{\prime}}\left[\phi_{0}(\mathbf{z})-\int_{\mathbf{z}_{1}, \mathbf{x}_{1}} G_{0}\left(\mathbf{x}_{1}, \mathbf{z}-\mathbf{z}_{1}\right) j\left(\mathbf{x}_{1}, \mathbf{z}_{1}\right)\right] G^{-1}\left(0, \mathbf{z}-\mathbf{z}^{\prime}\right)\left[\phi_{0}\left(\mathbf{z}^{\prime}\right)-\int_{\mathbf{z}_{2}, \mathbf{x}_{2}} G_{0}\left(\mathbf{x}_{2}, \mathbf{z}^{\prime}-\mathbf{z}_{2}\right) j\left(\mathbf{x}_{2}, \mathbf{z}_{2}\right)\right]
\end{aligned}
$$

For $j(\mathbf{r})$ and $\mathcal{H}_{p}\left[\phi_{0}(\mathbf{z})\right]=-v \int_{z} \cos \phi_{0}(z), \mathcal{W}\left[\phi_{0}(\mathbf{z}), 0\right]$ simply reduces to the Hamiltonian, Eq.2.11 localized on the pin, with the integrated out bulk degrees of freedom reflected in its long-range elasticity. On the other hand, for a finite $j(\mathbf{r})$, but strong pinning, on sufficiently long scales $e^{-\mathcal{H}_{p}\left[\phi_{0}(\mathbf{z})\right]}$ simply acts as a hard constraint $\phi_{0}(\mathbf{z})=0$, reducing $Z[j(\mathbf{r})]$ to the previously found result given in Eqs.B2, B3, B5.

In the pinned (commensurate) phase, a columnar defect pins a single vortex line, corresponding to a "confinement" of field $\phi_{0}(\mathbf{z})$ to a single minimum of the cosine and allowing us to approximate $\mathcal{H}_{p}\left[\phi_{0}(\mathbf{z})\right]=$ $-v \int_{\mathbf{z}} \cos \phi_{0}(\mathbf{z}) \approx$ const. $+\frac{1}{2} v \int_{\mathbf{z}} \phi_{0}(\mathbf{z})^{2}$ by a harmonic "spring". We can therefore integrate over $\phi_{0}(\mathbf{z})$ in Eq.B14, obtaining (up to an unimportant multiplicative constant):

$$
Z[j(\mathbf{r})]=e^{\frac{1}{2} \int_{\mathbf{r}, \mathbf{r}^{\prime}} j(\mathbf{r}) \Gamma_{\text {pinned }}\left[\mathbf{r}, \mathbf{r}^{\prime}\right] j\left(\mathbf{r}^{\prime}\right)} .
$$

with

$$
\begin{aligned}
\Gamma_{\text {pinned }}\left[\mathbf{r}, \mathbf{r}^{\prime}\right] & =\int_{\mathbf{z}_{1}, \mathbf{z}_{1}^{\prime}} G\left(\mathbf{x}, \mathbf{z}_{1}^{\prime}-\mathbf{z}\right) G^{-1}\left(0, \mathbf{z}_{1}-\mathbf{z}_{1}^{\prime}\right)\left[v \delta\left(\mathbf{z}_{1}-\mathbf{z}_{2}\right)+G^{-1}\left(0, \mathbf{z}_{1}-\mathbf{z}_{2}\right)\right]^{-1} G\left(\mathbf{x}^{\prime}, \mathbf{z}_{2}^{\prime}-\mathbf{z}^{\prime}\right) G^{-1}\left(0, \mathbf{z}_{2}-\mathbf{z}_{2}^{\prime}\right) \\
& +G\left(\mathbf{r}-\mathbf{r}^{\prime}\right)-\int_{\mathbf{z}_{1}, \mathbf{z}_{2}} G\left(\mathbf{x}, \mathbf{z}-\mathbf{z}_{1}\right) G^{-1}\left(0, \mathbf{z}_{1}-\mathbf{z}_{2}\right) G\left(\mathbf{x}^{\prime}, \mathbf{z}^{\prime}-\mathbf{z}_{2}\right)
\end{aligned}
$$

In Fourier space, this becomes 


$$
\begin{aligned}
\Gamma_{\text {pinned }}\left[\mathbf{q}, \mathbf{q}^{\prime}\right] & =(2 \pi)^{d_{z}} \delta^{d_{z}}\left(\mathbf{q}_{z}+\mathbf{q}_{z}^{\prime}\right)\left[(2 \pi)^{d_{x}} \delta^{d_{x}}\left(\mathbf{q}_{x}+\mathbf{q}_{x}^{\prime}\right) \tilde{G}\left(\mathbf{q}_{x}, \mathbf{q}_{z}\right)\right. \\
& \left.-\tilde{G}\left(\mathbf{q}_{x}, \mathbf{q}_{z}\right) \tilde{G}^{-1}\left(\mathbf{x}=0,-\mathbf{q}_{z}\right) \tilde{G}\left(\mathbf{q}_{x}^{\prime},-\mathbf{q}_{z}\right) \frac{v}{v+\tilde{G}^{-1}\left(\mathbf{x}=0, \mathbf{q}_{z}\right)}\right]
\end{aligned}
$$

For a system that is translationally invariant along $\mathbf{z}$, $\tilde{G}^{-1}\left(\mathbf{x}=0, \mathbf{q}_{z}\right)$ generically vanishes at long wavelengths $\mathbf{q}_{z} \rightarrow 0$, and the result reduces to that of a hard constraint, given in Eqs.B2, B3. In more detail for the $(1+1) \mathrm{D}$ vortex problem at hand, $\tilde{G}^{-1}\left(\mathbf{x}=0, \mathbf{q}_{z}\right)=$ $(a / 2 \pi)^{2} 2 \sqrt{K B}\left|q_{z}\right|$, showing that, as asserted in the main text, the crossover to the hard constraint happens on scales longer than the pinning length, $q_{z}^{-1} \gg \xi$.

\section{APPENDIX C: HILBERT TRANSFORM BASICS}

In this appendix, for completeness we summarize some of the basics of Hilbert transforms necessary to derive results in the main text and in the Appendixes.

Hilbert transform $\tilde{\phi}(y)=\mathrm{H}[\phi(x)]$ of a function $\phi(x)$ is defined by

$$
\mathrm{H}[\phi(x)]=\frac{1}{\pi} \mathrm{P} \int_{-\infty}^{\infty} d x \frac{\phi(x)}{x-y},
$$

where $\mathrm{P}$ stands for the principal value of the integral i.e., with the singular point $x=y$ excluded.

Hilbert transforms of standard functions can be usually computed by relating it to a contour integral in a complex plane. For example, Hilbert transform of $\sin x$ and $\cos x$ can be computed as real and imaginary parts of Hilbert transform of $e^{i x}$

$$
\begin{aligned}
\mathrm{H}\left[e^{i x}\right] & =\frac{1}{\pi} \mathrm{P} \int_{-\infty}^{\infty} d x \frac{e^{i x}}{x-y}, \\
& =i e^{i y}
\end{aligned}
$$

with last expression obtained easily by contour integration, taking advantage of analyticity of $e^{i x}$ in the upperhalf plane. Above result then leads to

$$
\begin{aligned}
\mathrm{H}[\sin x] & =\cos y, \\
\mathrm{H}[\cos x] & =-\sin y .
\end{aligned}
$$

More importantly for the problem of the vortex lattice at hand, we compute the Hilbert transform of a Lorentzian

$$
\begin{aligned}
\mathrm{H}\left[\frac{1}{x^{2}+1}\right] & =\frac{1}{\pi} \mathrm{P} \int_{-\infty}^{\infty} d x \frac{1}{x-y} \frac{1}{x^{2}+1}, \\
& =\frac{-y}{y^{2}+1}
\end{aligned}
$$

by noting that it is related a semi-circular contour integral over $C$ in the upper-half plane. Equivalently, it can be computed as (minus) the imaginary part of Hilbert transform of $\frac{x-i}{x^{2}+1}=\frac{1}{x+i}$, with the latter function analytic in the upper-half plane. As a side benefit the real part of $\mathrm{H}\left[\frac{1}{x+i}\right]$ gives

$$
\mathrm{H}\left[\frac{x}{x^{2}+1}\right]=\frac{1}{y^{2}+1} .
$$

\section{APPENDIX D: SOLITON SOLUTION OF THE SINE-HILBERT EQUATION}

In this appendix we verify that the soliton solution

$$
\phi_{s}(z)=-2 \operatorname{ArcTan} \frac{1}{z}
$$

indeed satisfies the Euler-Langrange integral equation

$$
\frac{1}{\pi} \int d z^{\prime} \frac{\phi_{s}(z)-\phi_{s}\left(z^{\prime}\right)}{\left(z-z^{\prime}\right)^{2}}+\sin \phi_{s}(z)=0
$$

where from now on, all the integrals are understood in the sense of a principal part, a physically dictated regularization. To this end, using a relation $\mathrm{P} \int \frac{d z^{\prime}}{\left(z-z^{\prime}\right)^{2}}=0$ and integrating by parts, we note that the sine-Hilbert equation can be rewritten as

$$
\begin{aligned}
-\partial_{z} \mathrm{H}\left[\phi_{s}\left(z^{\prime}\right)\right]+\sin \phi_{s}(z) & =0 \\
-\mathrm{H}\left[\partial_{z^{\prime}} \phi_{s}\left(z^{\prime}\right)\right]+\sin \phi_{s}(z) & =0 .
\end{aligned}
$$

Now using

$$
\partial_{z} \phi_{s}(z)=\frac{2}{z^{2}+1},
$$

and Hilbert transform relation from Appendix C, Eq.C7, we find

$$
\mathrm{H}\left[\partial_{z^{\prime}} \phi_{\left.\left(z^{\prime}\right)\right]}=\frac{-2 z}{z^{2}+1} .\right.
$$

Then calculating

$$
\begin{aligned}
\sin \phi_{s}(z) & =-\sin \left[2 \operatorname{ArcTan} \frac{1}{z}\right] \\
& =\frac{-2 z}{z^{2}+1}
\end{aligned}
$$

shows that indeed $\phi_{s}(z)$ satisfies the sine-Hilbert equation, Eq.D4. 


\section{APPENDIX E: SINGLE SOLITON ENERGY}

In this appendix we compute the energy $\hat{E}_{1}[\hat{h}]=$ $\hat{\mathcal{H}}_{0}\left[\phi_{s}(\hat{z})\right]$ of a single soliton,

$$
\phi_{s}(\hat{z})=-2 \operatorname{ArcTan} \frac{1}{\hat{z}-\hat{z}_{0}},
$$

for the sine-Hilbert model, defined by a Hamiltonian

$$
\hat{\mathcal{H}}_{0}=\hat{\mathcal{H}}_{0}^{e l}+\hat{\mathcal{H}}_{0}^{p},
$$

$$
\begin{aligned}
= & \frac{1}{4 \pi} \iint d \hat{z} d \hat{z}^{\prime}\left(\frac{\phi(\hat{z})-\phi\left(\hat{z}^{\prime}\right)-\hat{h}\left(\hat{z}-\hat{z}^{\prime}\right)}{\hat{z}-\hat{z}^{\prime}}\right)^{2} \\
& -\int d \hat{z} \cos \phi(\hat{z}),
\end{aligned}
$$

where all integrals are implicitly understood to range over the system size, with $-\hat{L} / 2<\hat{z}<\hat{L} / 2$. Expanding the square of the elastic part, $\hat{E}_{1}^{e l}=\hat{\mathcal{H}}_{0}^{e l}\left[\phi_{s}(\hat{z})\right]$, we find (to accuracy of $\mathcal{O}(1)$ for $\hat{L} \rightarrow \infty)$ :

$$
\begin{aligned}
\hat{E}_{1}^{e l} & =\frac{1}{2 \pi} \iint d \hat{z} d \hat{z}^{\prime}\left[\frac{\phi_{s}(\hat{z})^{2}-\phi_{s}(\hat{z}) \phi_{s}\left(\hat{z}^{\prime}\right)}{\left(\hat{z}-\hat{z}^{\prime}\right)^{2}}-\hat{h} \frac{\phi_{s}(\hat{z})-\phi_{s}\left(\hat{z}^{\prime}\right)}{\hat{z}-\hat{z}^{\prime}}\right]+\frac{1}{4 \pi} \hat{h}^{2} \hat{L}^{2}, \\
& =-\frac{1}{2} \int d \hat{z} \phi_{s}(\hat{z}) \mathrm{H}\left[\partial_{\hat{z}} \phi_{s}(\hat{z})\right]-\hat{h} \int d \hat{z} \mathrm{H}\left[\phi_{s}(\hat{z})\right]+\frac{1}{4 \pi} \hat{h}^{2} \hat{L}^{2} .
\end{aligned}
$$

These integrals can be computed utilizing Hilbert transforms worked out in Appendix C. Using Eq.C7, the first term, $\hat{E}_{1 a}^{e l}$ can be integrated by parts

$$
\begin{aligned}
\hat{E}_{1 a}^{e l} & =-\frac{1}{2} \int d \hat{z} \phi_{s}(\hat{z}) \mathrm{H}\left[\partial_{\hat{z}} \phi_{s}(\hat{z})\right], \\
& =-2 \int d \hat{z} \frac{\hat{z}}{\hat{z}^{2}+1} \operatorname{ArcTan} \frac{1}{\hat{z}}, \\
& =2 \pi \ln \frac{\hat{L}}{4} .
\end{aligned}
$$

Less formally, this elastic contribution can be computed by going back to the expression

$$
\hat{E}_{1 a}^{e l}=\frac{1}{4 \pi} \iint d \hat{z} d \hat{z}^{\prime}\left(\frac{\phi_{s}(\hat{z})-\phi_{s}\left(\hat{z}^{\prime}\right)}{\hat{z}-\hat{z}^{\prime}}\right)^{2},
$$

and noting that because $\phi_{s}(\hat{z})$ vanishes for $\hat{z} \gtrsim 1$ and equals $2 \pi$ for $\hat{z} \lesssim-1$, finite contributions to the elastic energy arise only from regions $\left(\hat{z} \lesssim-1, \hat{z}^{\prime} \gtrsim 1\right)$ and $(\hat{z} \gtrsim$ $\left.1, \hat{z}^{\prime} \lesssim-1\right)$. To accuracy of $\mathcal{O}(1)$, this reduces the soliton elastic energy to

$$
\begin{aligned}
\hat{E}_{1 a}^{e l} & \approx \frac{1}{\pi} \int_{-\hat{L} / 2}^{-1} d \hat{z} \int_{1}^{\hat{L} / 2} d \hat{z}^{\prime} \frac{(2 \pi)^{2}}{\left(\hat{z}-\hat{z}^{\prime}\right)^{2}} \\
& \approx 2 \pi \ln \frac{\hat{L}}{4}
\end{aligned}
$$

in agreement with the more formal analysis above.

The second contribution to $\hat{E}_{1}^{e l}$ in Eq.E4 can also be computed by integrating by parts and noting that $\mathrm{H}\left[\phi_{s}(\hat{z})\right]$ (that can be explicitly computed giving $\left.\mathrm{H}\left[\phi_{s}(\hat{z})\right]=-\ln \left[\frac{\hat{z}^{2}+1}{(\hat{L} / 2)^{2}+1}\right]\right)$ vanishes at the boundaries of the system, $\hat{z}= \pm \hat{L} / 2$

$$
\hat{E}_{1 b}^{e l}=-\hat{h} \int_{-\hat{L} / 2}^{\hat{L} / 2} d \hat{z} \mathrm{H}\left[\phi_{s}(\hat{z})\right],
$$

$$
\begin{aligned}
& =\hat{h} \int_{-\hat{L} / 2}^{\hat{L} / 2} d \hat{z} \hat{z} \mathrm{H}\left[\partial_{\hat{z}} \phi_{s}(\hat{z})\right], \\
& =-\hat{h}(2 \hat{L}-2 \pi) .
\end{aligned}
$$

A single soliton pinning contribution $\hat{E}_{1}^{p}=\hat{\mathcal{H}}_{0}^{p}\left[\phi_{s}(\hat{z})\right]$ is also straightforward to calculate using solution $\phi_{s}(\hat{z})$, Eq.E1. We find

$$
\begin{aligned}
\hat{E}_{1}^{p} & =-\int_{-\hat{L} / 2}^{\hat{L} / 2} d \hat{z} \cos \phi_{s}(\hat{z}), \\
& =-\int_{-\hat{L} / 2}^{\hat{L} / 2} d \hat{z}\left(1-2 \sin ^{2}\left(\phi_{s} / 2\right)\right), \\
& =-\int_{-\hat{L} / 2}^{\hat{L} / 2} d \hat{z}\left(1-\frac{2}{\hat{z}^{2}+1}\right), \\
& =-\hat{L}+2 \pi .
\end{aligned}
$$

Combining above contributions inside Eq.E2, we obtain the expression for a single soliton dimensionless energy

$$
\begin{aligned}
\hat{E}_{1} & =\frac{1}{4 \pi} \hat{h}^{2} \hat{L}^{2}-\hat{L}+2 \pi \ln \frac{e \hat{L}}{4}-\hat{h}(2 \hat{L}-2 \pi), \\
& \approx \frac{1}{4 \pi} \hat{h}^{2} \hat{L}^{2}-\hat{L}+2 \pi \ln \hat{L}-2 \hat{h} \hat{L}
\end{aligned}
$$

used in the main text.

\section{APPENDIX F: TWO-SOLITON ENERGY: INTERACTION}

In this appendix we give a few technical details for the computation of the soliton interaction energy. For two far-separated solitons (of interest in a dilute soliton 
approximation, valid for $\left.h \ll h_{c 2}\right)$ with $\left|\hat{z}_{1}-\hat{z}_{2}\right| \gg 1$, we can approximate the exact two-soliton solution by a sum of two one-soliton solutions

$$
\phi_{2 s}\left(\hat{z}_{1}, \hat{z}_{2}\right) \approx \phi_{s}\left(\hat{z}_{1}\right)+\phi_{s}\left(\hat{z}_{2}\right)
$$

$$
\begin{aligned}
\hat{E}_{2}\left(\hat{z}_{1}, \hat{z}_{2}\right) & \approx \hat{\mathcal{H}}_{0}\left[\phi_{s 1}+\phi_{s 2}\right] \\
& =\frac{1}{4 \pi} \iint d \hat{z} d \hat{z}^{\prime}\left(\frac{\phi_{s 1}(\hat{z})+\phi_{s 2}(\hat{z})-\phi_{s 1}\left(\hat{z}^{\prime}\right)-\phi_{s 2}\left(\hat{z}^{\prime}\right)-\hat{h}\left(\hat{z}-\hat{z}^{\prime}\right)}{\hat{z}-\hat{z}^{\prime}}\right)^{2}-\int d \hat{z} \cos \left[\phi_{s 1}(\hat{z})+\phi_{s 2}(\hat{z})\right] \\
& =\hat{E}_{C}+2 \hat{E}_{s 1}+V_{s}\left(\hat{z}_{1}-\hat{z}_{2}\right),
\end{aligned}
$$

that consists of the zero-soliton contribution $\hat{E}_{C}$, Eq.3.20, two one-soliton contributions $\hat{E}_{s 1}$, and soliton interaction

$$
V_{s}\left(\hat{z}_{1}-\hat{z}_{2}\right)=V_{s}^{A}\left(\hat{z}_{1}-\hat{z}_{2}\right)+V_{s}^{B}\left(\hat{z}_{1}-\hat{z}_{2}\right) \text { given by }
$$

The two-soliton interaction is then determined by twosoliton energy

,

$$
\begin{aligned}
V_{s}^{A}\left(\hat{z}_{1}-\hat{z}_{2}\right) & =\frac{1}{\pi} \iint d \hat{z} d \hat{z}^{\prime} \frac{\phi_{s 1}(\hat{z}) \phi_{s 2}(\hat{z})-\phi_{s 1}(\hat{z}) \phi_{s 2}\left(\hat{z}^{\prime}\right)}{\left(\hat{z}-\hat{z}^{\prime}\right)^{2}} \\
V_{s}^{B}\left(\hat{z}_{1}-\hat{z}_{2}\right) & =\int d \hat{z}\left[1-\cos \phi_{s 1}(\hat{z}) \cos \phi_{s 2}(\hat{z})+\sin \phi_{s 1}(\hat{z}) \sin \phi_{s 2}(\hat{z})\right]
\end{aligned}
$$

Manipulations similar to those for the computation of tion gives a single soliton energy give

$$
\begin{aligned}
V_{s}^{A}\left(\hat{z}_{1}-\hat{z}_{2}\right) & \approx-\int d \hat{z} \phi_{s 1}(\hat{z}) \mathrm{H}\left[\partial_{\hat{z}} \phi_{s 2}(\hat{z})\right] \\
& \approx-4 \int d \hat{z} \frac{\hat{z}-\hat{z}_{2}}{\left(\hat{z}-\hat{z}_{2}\right)^{2}+1} \operatorname{ArcTan} \frac{1}{\hat{z}-\hat{z}_{1}} \\
& =4 \pi \ln \frac{\hat{L}}{2}-2 \int_{-\infty}^{\infty} d \hat{z} \frac{\ln \left[\left(\hat{z}-\hat{z}_{2}\right)^{2}+1\right]}{\left(\hat{z}-\hat{z}_{1}\right)^{2}+1} \\
& =4 \pi \ln \frac{\hat{L}}{2}-2 \pi \ln \left[\left(\hat{z}_{1}-\hat{z}_{2}\right)^{2}+4\right]
\end{aligned}
$$

with a simplifying approximation above valid for $\left|z_{1,2}\right| \ll$ $L / 2 \rightarrow \infty$. Similarly, a straightforward contour integra-

$$
\begin{aligned}
& (\mathrm{F} 7)^{V_{s}^{B}}\left(\hat{z}_{1}-\hat{z}_{2}\right)=2 \int_{-\infty}^{\infty} d \hat{z} \frac{\left(2 \hat{z}-\hat{z}_{1}-\hat{z}_{2}\right)^{2}}{\left(\left(\hat{z}-\hat{z}_{1}\right)^{2}+1\right)\left(\left(\hat{z}-\hat{z}_{2}\right)^{2}+1\right)}, \\
& =4 \pi \text {, }
\end{aligned}
$$

that, together with Eq.F10 gives the soliton interaction

$$
V_{s}(\hat{z})=2 \pi \ln \left[\frac{(\hat{L} / 2)^{2}}{\hat{z}^{2}+4}\right]+4 \pi
$$

used in the main text.
${ }^{1}$ M .Tinkham, Introduction to Superconductivity, (Krieger Publishing Company, 1980).

${ }^{2}$ G. Blatter, M.V. Feigel'man, V.B. Geshkenbein, A.I. Larkin, and V.M. Vinokur, Rev. Mod. Phys. 66, 1125 (1994).

3 T. Nattermann and S. Scheidl, Adv. Phys. 49, 607 (2000).

${ }^{4}$ D. S. Fisher, M. P. A. Fisher, and D. A. Huse, Phys. Rev. $B$ 43, 130 (1991), and references therein.

${ }^{5}$ D. A. Huse and L. Radzihovsky, in Proceedings of 1993 Altenberg Summer School, Fundamental Problems in Sta- tistical Mechanics VIII, edited by H. van Beijeren and M. H. Ernst (Elsevier, Netherlands).

6 O. Narayan and D.S. Fisher, Phys. Rev. B 46, 11520 (1992). A.E. Koshelev and V.M. Vinokur, Phys. Rev. Lett. 73, 3580 (1994). T. Giamarchi and P. Le Doussal, Phys. Rev. Lett. 76, 3408 (1996); Phys. Rev. B 57, 11356 (1998). L. Balents, M.C. Marchetti and L. Radzihovsky, Phys. Rev. Lett. 78, 751 (1997); Phys. Rev. B 57, 7705 (1998). S. Scheidl and V.M. Vinokur, Phys. Rev. B 57, 13800 (1998), and references therein. 
7 G. Eilenberger, Phys. Rev. 164, 628 (1967).

8 D.S. Fisher, Phys. Rev. B 22, 1190 (1980).

9 D.R. Nelson, Phys. Rev. Lett. 60, 1973 (1988); D.R. Nelson and S. Seung, Phys. Rev. B 39, 9153 (1989).

10 P.L. Gammel, L.F. Scheemeyer, J.V. Waszczak, and D.J. Bishop, Phys. Rev. Lett. 61, 1666 (1988). R. Cubitt, E. M. Forgan, G. Yang, S. L. Lee, D. M. Paul, H. A. Mook, M. Yethiraj, P. H. Kes, T. W. Li, A. A. Menovsky, Z. Tarnawski, and K. Mortensen, Nature 365, 407 (1993). E. Zeldov, D. Majer, M. Konczykowski, V. B. Geshkenbein, V. M. Vinokur, and H. Shtrikman, Nature 375, 373 (1995); E. Zeldov, et. al. Nature 382, 791 (1996).

11 A. Larkin, Sov. Phys. JETP 31, 784 (1970); A.I. Larkin and Y.N. Ovchinnikov, Sov. Phys. JETP 38, 854 (1974).

${ }^{12}$ Y. Imry and S. K. Ma, Phys. Rev. Lett. 35, 1399 (1975).

13 M. P. A. Fisher, Phys. Rev. Lett. 62, 1415 (1989); J. L. Cardy and S. Ostlund, Phys. Rev. B 25, 6899 (1982).

14 R.H. Koch et al., Phys. Rev. Lett. 63, 1511 (1989).

15 S. F. Edwards and P. W. Anderson, J. Phys. F 5, 4841 (1975).

${ }^{16}$ L. Civale, A. D. Marwick, T. K. Worthington, M. A. Kirk, J. R. Thompson, L. Krusin-Elbaum, Y. Sum, J. R. Clem and F. Holtzberg, Phys. Rev. Lett. 67, 648 (1991).

17 M. P. A. Fisher and D. H. Lee, Phys. Rev. B 39, 2756 (1989).

18 D. R. Nelson and V. M. Vinokur, Phys. Rev. Lett. 68, 2398 (1992); Phys. Rev. B. 48, 13060 (1993).

19 M. P. A. Fisher, P. B. Weichman, G. Grinstein, and D. S. Fisher, Phys. Rev. B 40, 546 (1989).

20 R. C. Budhani, M. Suenga, and S. H. Liou, Phys. Rev. Lett. 69, 3816 (1992); W. Jiang, N. -C. Yeh, D. S. Reed, U. Kriplani, D. A. Beam, M. Konczykowski, T. A. Tombrello, and F. Holtzberg, Phys. Rev. Lett. 72, 550 (1994); S. A. Grigera, E. Morre', E. Osquiguil, C. Balseiro, G. Nieva, and F. de la Cruz, Phys. Rev. Lett. 81, 2348 (1998).

21 J. Lidmar and M. Wallin, Europhys. Lett. 47, 494 (1999).

22 L. Balents, Europhys. Lett. 24, 489 (1993).

23 T. Hwa, D. R. Nelson, and V. M. Vinokur, Phys. Rev. B 48, 1167 (1993).

24 D. R. Nelson and L. Radzihovsky, Phys. Rev. B 54, R6845 (1996).

25 B. I. Shklovskii and A. L. Efros, Electronic Properties of Doped Semiconductors (Springer, New York, 1984).

26 U. C. Tauber and D. R. Nelson, Phys. Rep. 289, 157 (1997); C. Wengel and U. C. Tauber, Phys. Rev. Lett. 78, 4845 (1997).

27 D. S. Fisher, Phys. Rev. Lett. 78, 1964 (1997).

28 T. Giamarchi and P. Le Doussal, Phys. Rev. Lett. 72, 1530 (1994); Phys. Rev. B 52, 1242 (1995).

29 T. Nattermann, Phys. Rev. Lett. 64, 2454 (1990).

30 S.E. Korshunov, Phys. Rev. B 48; 3969 (1993)

31 D. S. Fisher, Phys. Rev. B 31, 7233 (1985).

${ }^{32}$ W. K. Kwok, et al., Phys. Rev. Lett. 72, 1088 (1994).

33 S. Debrion, W. R. White, A. Kapitulnik, and M. R. Beasley, Phys. Rev. B 49 (1994).

34 S. Raedts, A. V. Silhanek, M. J. Van Bael, V. V. Moshchalkov, Phys. Rev. B 70, 024509 (2004).

${ }^{35}$ W. Hofstetter, I. Affleck, D. R. Nelson, and U. Schollwock, Europhys. Lett. 66, 178 (2004); I. Affleck, W. Hofstetter, D. R. Nelson, and U. Schollwock, Journal of Stat. Mech.: Theory and Experiment, P10003 (2004).

36 L. Radzihovsky, Phys. Rev. Lett. 74, 4923 (1995).

37 F. D. M. Haldane, Phys. Rev. Lett. 45, 1358 (1980); 47, 1840 (1981).
38 C. L. Kane and M. P. A. Fisher, Phys. Rev. Lett. 68, 1220 (1992); C. L. Kane and M. P. A. Fisher, Phys. Rev. B 46, 15233 (1992);

39 P. Fendley, A.W.W. Ludwig, and H. Saleur, Phys. Rev. B 52, 8934 (1995); Phys. Rev. Lett. 743005 (1995).

40 A. Polkovnikov, Y. Kafri and D. R. Nelson, condmat/0409520.

41 J.V. José, L.P. Kadanoff, S.Kirkpatrick and D.R. Nelson, Phys. Rev. B 16, 1217 (1977); 17, 1477 (1978) (Erratum).

42 V.L. Pokrovsky, A. L. Talapov and P. Bak, in Solitons, edited by S.E. Trullinger, V. E. Zakharov and V. L. Pokrovsky (North Holland, Amsterdam, 1986), Chap. 3, pp. $71-127$.

43 S.N. Coppersmith, D.S. Fisher, B.I. Halperin, P.A. Lee, and W.F. Brinkman, Phys. Rev. B 25, 349 (1982).

44 S. Chakravarty, G. Ingold, S. Kivelson, and A. Luther, Phys. Rev. Lett. 56, 2303 (1986); S. Chakravarty, G. Ingold, S. Kivelson, and G. Zimanyi, Phys. Rev. B 37, 3283 (1988); M. P. A. Fisher, Phys. Rev. Lett. 57, 885 (1986).

45 A. J. Leggett, S. Chakravarty, A. T. Dorsey, M. P. A. Fisher, A. Garg, and W. Zwerger, Rev. Mod. Phys. 59, 1 (1987).

46 J. M. Kosterlitz, Phys. Rev. Lett. 37, 1577 (1976).

47 P. W. Anderson, G. Yuval, and D. R. Hamann, Phys. Rev. $B$ 1, 4464 (1970).

48 There is no transition in finite system, as nonanalyticity can only appear in thermodynamic limit. Given that, in $L \rightarrow \infty$ limit the critical value $h_{c 1}$ for the dimensionless transverse tilting field is driven to 0 , one concludes that indeed for one pin there is no phase transition. However, as in analogous problems, e.g., Kondo ${ }^{47}$, we imagine that in a physical system there is a finite dilute (and therefore noninteracting) concentration of columnar pins, which will prevent $h_{c 1}$ from being driven to 0 and will exhibit a true CI tilting transition studied here.

49 E.H. Brandt and U. Essman, Phys. Status Solidi B 144, 13 (1987). A. Houghton, R. A. Pelcovits and A. Sudbo, Phys. Rev. B 40, 6763 (1989). D.S. Fisher, in Phenomenology and Applications of High-Temperature Superconductors, K.S. Bedell et al., eds. (Addison-Wesley, 1992), p. 287.

50 Elastic moduli $K$ and $B$ can be derived from the GinzburgLandau theory and for finite-range interactions (set by the London penetration length $\lambda$ ) are wavevector dependent on scales shorter than $\lambda$, and become dispersionless only on scales longer than $\lambda$, i.e., for $n_{0} \lambda \ll 1$. For simplicity we will ignore this inessential complication.

51 Recent experimental progress in ultra-cold trapped atoms demonstrated ability to tune interactions via a magnetic field by utilizing a proximity to a Feshbach resonance. See, e.g., C. A. Regal, et al., Phys. Rev. Lett. 92, 040403 (2004). E. Timmermans, K. Furuya, P. W. Milonni, and A. K. Kerman, Phys. Lett. A 285, 228 (2001).

52 General arguments suggest that the effective Luttinger parameter $g$ (proportional to $1 / \sqrt{K B}$ ) is constrained by 1 from above, corresponding to a universal, noninteracting fermion limit ${ }^{42,43,53,54}$ reached in the dilute vortex gas near $H_{c 1}$. However, at high densities, such that vortex spacing falls below the London penetration length elastic moduli become wavevector dependent. Furthermore, at higher densities and lower temperature, fluctuation (Helfrich) contribution ${ }^{42,43}$ to compressional modulus becomes subdominant. Hence in this regime the Luttinger parameter can exceed 1, allowing for the roughening (thermal depinning) transition at $g=1 .{ }^{35}$ 
${ }_{53}$ M. D. Girardeau, J. Math. Phys. (N.Y.) 1, 516 (1960).

54 E. H. Lieb and W. Liniger, Phys. Rev. 130, 1605, (1963); E. H. Lieb, ibid 130, 1616 (1963).

55 J. M. Kosterlitz and D. J. Thouless, J. Phys. C 6, 1181 (1973).

56 It is important here to distinguish the problem of an applied transverse field $H_{\perp}$ considered here, from the problem of a pure rotation of a vortex lattice. The former case is well-described by a Hamiltonian in Eq.2.1, with a longitudinal component of the flux density (taken along $\hat{\mathbf{z}}$ ) held fixed, and the total magnetic field $\sqrt{H_{z}^{2}+H_{\perp}^{2}}$ increasing with applied $H_{\perp}$. Consistently, in this case the elastic energy is minimized by $u(x, z)=z H_{\perp} / H_{z}=$ $z \tan \theta$. In contrast, a pure rotation of the vortex lattice (in the $x-z$ plane) by angle $\theta$, keeps the true vortex density (defined as the inverse of vortex spacing transverse to vortex average orientation) proportional to $\sqrt{H_{z}^{2}+H_{\perp}^{2}}$ fixed. By necessity such rotation decreases $x$-projected vortex spacing, corresponding to a decrease in the $x$-projected vortex density by amount $-n_{0} \partial_{x} u=$ $-n_{0}(1-\cos \theta)<0$. As is well-known in the context of smectic liquid crystals, ${ }^{57}$, to capture full rotational invariance, the compressional term must be modified. The appropriate elastic Hamiltonian in this case is given by $H_{e l}^{r}=\frac{1}{2} \int d x d z\left[K\left(\partial_{z} u-h_{r}\right)^{2}+B\left(\partial_{x} u-\frac{1}{2}(\nabla u)^{2}\right)^{2}\right]$, with $h_{r}=\sin \theta=H_{\perp} / \sqrt{H_{z}^{2}+H_{\perp}^{2}}$ and is minimized by $u_{0}(x, z)=x(\cos \theta-1)-z \sin \theta$, that describes a pure rotation of the vortex lattice in the $x-z$ plane.

57 P. G. de Gennes and J. Prost, The Physics of Liquid Crystals, (Clarendon Press, Oxford, 1993).

58 D. S. Fisher, K. Dahmen, S. Ramanathan, and Y. BenZion, Phys. Rev. Lett. 78, 4885 (1997).

${ }^{59}$ P. M. Santini, M. J. Ablowitz and A. S. Fokas, J. Math. Phys. 28 10, (1987).

60 R. Peierls, Proc. Phys. Soc. 52, 34 (1940); F. R. N. Nabarro, Proc. Phys. Soc. 59, 256 (1947).

61 Soliton proliferation CI transition at $h=h_{c 1}$ is closely analogous to the transition at $H_{c 1}$ from the Meissner state to the vortex state in type II superconductors. The trans- verse field $h_{c 2}$, at which solitons form a dense overlapping array with $n_{s} \approx 1 / \xi$ corresponding to a nearly perfect vortex alignment with an external field (i.e., absence of transverse diamagnetism), then corresponds to the uppercritical $H_{c 2}$ field at which the system asymptotes to the normal state. In this identification, the bulk $h_{c}$ field corresponds to the thermodynamic $H_{c}$ field of a type-II superconductor, beyond which the energy of a bulk superconducting Meissner state exceeds that of the normal state.

62 We expect that in contrast to systems in which solitons interact via a short-range potential (as in e.g., sine-Gordon model), here, because of logarithmic soliton interaction we expect the approximation of a multi-soliton solution by a sum of one-soliton solutions to be less accurate. Unfortunately, it is quite challenging to go beyond this approximation analytically. Nevertheless, we expect that all qualitative (and some quantitative) predictions made here will remain unchanged.

${ }^{63}$ In the independent soliton approximation used here this cancellation is not complete. Although we have not shown this explicitly, from experience with the analogous calculation for the sine-Gordon model and physical considerations it is clear that if the proper multi-soliton solution is used, here too the total $N_{s}$-soliton energy $E_{N s}$ should approach 0 in the dense soliton limit, $h \rightarrow h_{c 2}$, as the fully incommensurate state, $u_{C}=h z$ is approached.

${ }^{64}$ Our definition of the pinning number $M_{p}$ should not be confused with the related quantitity $N_{p}$ made in Ref. 35 . Although the two measures of the pinning influence of the columnar deffect are different, they nearly agree in the $h \gg$ $h_{c 1}$ limit.

65 K.G. Wilson and J. Kogut, Phys. Rep. 12, 75 (1974).

66 Absence of graphical corrections (renormalization) to $\sqrt{B K}$ is guaranteed by the long-range nature (nonanalytic form $|q|$ ) of the corresponding 1D vortex lattice elasticity. This property results in the exactly vertical renormalization-group flows of $v(\ell)$ in the $v-\sqrt{B K}$ plane, as for example found by Kane and Fisher ${ }^{38}$ 